\title{
On the macroscopic-mesoscopic mixture of a magnetorheological fluid
}

\author{
By Kuo-Ching Chen* \\ Institute of Applied Mechanics, National Taiwan University, \\ Taipei, Taiwan, Republic of China
}

This paper is concerned with the modelling of a magnetorheological (MR) fluid in the presence of an applied magnetic field as a twofolded mixture - a macroscopic fluid continuum and mesoscopic multi-solid continua. By assigning to each solid particle a vectorial mesoscopic variable, which is defined as the nearest relative position vector with respect to other particles, the solid medium of the MR fluid is further treated as a mixture consisting of different components, specified by these mesoscopic variables. The treatment of multi-solid continua is similar to that in the mesoscopic theory of liquid crystals. However, the key difference lies in the fact that the time-discontinuity of the defined vectorial mesoscopic variable will give rise to a 'pseudo' chemical reaction in the solid continuum. The equation of the phenomenological mesoscopic distribution function of the solid continuum then has an additional production term from the pseudo chemical reaction, analogous to the collision term appearing in the Boltzmann equation. The mesoscopic and macroscopic balance equations are then derived and by assuming the special constitutive relations the macroscopic equation for the second moment of the distribution function can be obtained.

Keywords: mesoscopic theory; magnetorheological fluids; solid-fluid mixture; balance equations; distribution function

\section{Introduction}

In the past two decades magnetorheological (MR) fluids or their electric analogues, electrorheological fluids (Block \& Kelly 1988; Ashour et al. 1996), have attracted much attention in the academic and industrial areas due not only to their potential applications in the semi-active control but also to their novel physical phenomena, e.g. phase transition, magnetic-dependent yield stress, and two longitudinal elastic waves (Nahmad-Molinari et al. 1999). An MR fluid is composed of a non-magnetized carrying fluid and suspended magnetizable particles of diamagnetic nature, the latter of which have uniform distribution in the absence of the applied magnetic field. When one turns on a magnetic field, the magnetic particles will almost instantaneously be magnetized and aggregate to form a stable chain-like or column-like structure along the direction of the field. This magnetic-dependent structure can be modified or destroyed by changing the flowing conditions such as applying a sufficiently strong shear force.

*kcc@spring.iam.ntu.edu.tw 
Hence, an MR material is a paradigm that internal structure can be formed or destroyed by the arrangement of the particles upon the action of applied fields.

In this paper, we propose a macroscopic-mesoscopic approach to investigate the behaviour of MR materials. Firstly, an MR material is modelled as a macroscopic mixture composed of a solid continuum, characterized by the arrangement of magnetizable particles, and a fluid continuum, represented as the non-magnetized carrying fluid. In the modelling approach, we assign to each particle of the solid continuum a vector $l$, the nearest relative positive vector among particles, to delineate the arrangement of the particles. The arrangement can be influenced by the magnitude of the magnetic field and the flow condition of the MR fluid. The vector $\boldsymbol{l}$ can be regarded as a continuous index so that the solid continuum can be further treated as a mesoscopic mixture of different components with individual indices $\boldsymbol{l}$ 's or mesoscopic variables $\boldsymbol{l}$ 's. The concept of a continuous index was also proposed by Faria (2001) and used by Faria \& Hutter (2002), who name it 'continuous diversity', to formulate the global and local master equations and jump conditions for chemically reacting mixtures of micro-structured media.

The concept of decomposition of a continuum into different components is called the mesoscopic concept, which originally was proposed by Blenk et al. (1991a,b, 1992) and Blenk \& Muschik (1991) in the theory of liquid crystals, where the liquid crystals were modelled as a mixture composed of different continua with different microscopic orientations of the molecules. To be explicit, instead of the macroscopic continuum level and the detailed microscopic description, the mesoscopic concept is to enlarge the domain of the field quantities by introducing the statistical distribution function of some specific property of the material (Papenfuss 2000). This concept has been successfully adopted in the modelling of liquid crystals (Muschik et al. 1995, 2000, 2004; Muschik \& Su 1997) and in materials with microcracks (Ván et al. 2000; Papenfuss et al. 2003). Potential applications definitely deserve further studies.

In the following, a preliminary framework of the macroscopic solid and fluid mixture for the modelling of MR materials is addressed in $\S 2$. In §3, the solid continuum is further regrouped as a mesoscopic mixture distinguished by a newly defined vector field, which bears a similar role as that of the microscopic director in the mesoscopic theory of liquid crystals (Papenfuss 2000). Section 4 presents the mesoscopic balance equations for the solid continuum and also introduces a new finding called the 'pseudo chemical reaction', which comes from the property of discontinuity in time for the vector $\boldsymbol{l}$. Section 5 gives the macroscopic field quantities and balance equations from the summation and integration of those of individual components of the whole mixture. The specific second moment equations for the mesoscopic distribution are discussed in $\S 6$ by the specification of the mesoscopic constitutive functions. Finally, general conclusions are given in $\$ 7$.

\section{Macroscopic solid and fluid mixture}

The concept of a general mixture theory is to allow the different continua to occupy a common physical space (Bowen 1976; Hutter \& Jöhnk 2004). For MR materials, Chen \& Yeh (2002a) modelled them macroscopically in the presence of a magnetic field as a mixture composed of a fluid continuum and an equivalent 
solid continuum. Let $\boldsymbol{X}^{\mathrm{F}}$ and $\boldsymbol{X}^{\mathrm{S}}$ be the material points and $\boldsymbol{x}^{\mathrm{F}}$ and $\boldsymbol{x}^{\mathrm{S}}$ denote the motions of the fluid and solid continua,

$$
\boldsymbol{x}^{\mathrm{F}}=\boldsymbol{x}^{\mathrm{F}}\left(\boldsymbol{X}^{\mathrm{F}}, t\right), \quad \boldsymbol{x}^{\mathrm{S}}=\boldsymbol{x}^{\mathrm{S}}\left(\boldsymbol{X}^{\mathrm{S}}, t\right),
$$

where the superscripts $\mathrm{F}$ and $\mathrm{S}$ represent the fluid and the solid. Since at time $t$, two material points of the different continua meet at the same point $\boldsymbol{x}$, we have

$$
\boldsymbol{x}=\boldsymbol{x}^{\mathrm{F}}=\boldsymbol{x}^{\mathrm{S}} \text {. }
$$

The different material points of two continua will give rise to different material time derivatives, which are the time derivatives keeping the respective material points fixed. The velocity vectors $\boldsymbol{v}^{\mathrm{F}}$ and $\boldsymbol{v}^{\mathrm{S}}$ of the two continua at time $t$ should be given as

$$
\boldsymbol{v}^{\mathrm{F}}=\frac{\mathrm{d}_{\mathrm{F}}}{\mathrm{d} t} \boldsymbol{x}^{\mathrm{F}}, \quad \boldsymbol{v}^{\mathrm{S}}=\frac{\mathrm{d}_{\mathrm{S}}}{\mathrm{d} t} \boldsymbol{x}^{\mathrm{S}},
$$

where the notations $\mathrm{d}_{\mathrm{F}} / \mathrm{d} t$ and $\mathrm{d}_{\mathrm{S}} / \mathrm{d} t$, respectively, designate the material time derivatives with $\boldsymbol{X}^{\mathrm{F}}$ and $\boldsymbol{X}^{\mathrm{S}}$ fixed. For the mixture the mean velocity or the velocity of the centre of mass at $(\boldsymbol{x}, t)$ is written as

$$
\boldsymbol{v}=\frac{\mathrm{d}}{\mathrm{d} t} \boldsymbol{x}=\frac{1}{\rho}\left(\rho^{\mathrm{F}} \boldsymbol{v}^{\mathrm{F}}+\rho^{\mathrm{S}} \boldsymbol{v}^{\mathrm{S}}\right),
$$

in which $\rho^{\mathrm{F}}, \rho^{\mathrm{S}}$ and $\rho\left(=\rho^{\mathrm{F}}+\rho^{\mathrm{S}}\right)$ denote the densities of the fluid, of the solid and of the mixture. We can further define the diffusion velocities for each continuum and the relative velocity vector of the fluid with respect to the solid as

$$
\overline{\boldsymbol{v}}^{\mathrm{F}}=\boldsymbol{v}^{\mathrm{F}}-\boldsymbol{v}, \quad \overline{\boldsymbol{v}}^{\mathrm{S}}=\boldsymbol{v}^{\mathrm{S}}-\boldsymbol{v}, \quad \boldsymbol{v}^{\mathrm{R}}=\boldsymbol{v}^{\mathrm{F}}-\boldsymbol{v}^{\mathrm{S}} .
$$

By using equation (2.4), one can transform the material time derivatives, $\mathrm{d}_{\mathrm{F}} / \mathrm{d} t$ and $\mathrm{d}_{\mathrm{S}} / \mathrm{d} t$, to a more useful material time derivative $\mathrm{d} / \mathrm{d} t$ following the barycentric motion as

$$
\begin{aligned}
& \frac{\mathrm{d}_{\mathrm{F}}}{\mathrm{d} t}=\frac{\mathrm{d}}{\mathrm{d} t}+\overline{\boldsymbol{v}}^{\mathrm{F}} \cdot \nabla=\frac{\mathrm{d}}{\mathrm{d} t}+\frac{\rho^{\mathrm{S}} \boldsymbol{v}^{\mathrm{R}}}{\rho} \cdot \nabla, \\
& \frac{\mathrm{d}_{\mathrm{S}}}{\mathrm{d} t}=\frac{\mathrm{d}}{\mathrm{d} t}+\overline{\boldsymbol{v}}^{\mathrm{S}} \cdot \nabla=\frac{\mathrm{d}}{\mathrm{d} t}-\frac{\rho^{\mathrm{F}} \boldsymbol{v}^{\mathrm{R}}}{\rho} \cdot \nabla .
\end{aligned}
$$

With no chemical reaction between the solid and the fluid, we recapitulate the macroscopic balance equations for the fluid and solid continua of MR materials as follows.

(i) Balances of mass:

$$
\frac{\partial \rho^{\mathrm{F}}}{\partial t}+\nabla \cdot\left(\rho^{\mathrm{F}} \boldsymbol{v}^{\mathrm{F}}\right)=0, \quad \frac{\partial \rho^{\mathrm{S}}}{\partial t}+\nabla \cdot\left(\rho^{\mathrm{S}} \boldsymbol{v}^{\mathrm{S}}\right)=0 .
$$

(ii) Balances of linear momentum:

$$
\begin{aligned}
& \rho^{\mathrm{F}} \frac{\mathrm{d}_{\mathrm{F}} \boldsymbol{v}^{\mathrm{F}}}{\mathrm{d} t}=\nabla \cdot \mathrm{t}^{\mathrm{F}}+\boldsymbol{P}^{\mathrm{F}}+\rho^{\mathrm{F}} \boldsymbol{f}^{\mathrm{F}}, \\
& \rho^{\mathrm{S}} \frac{\mathrm{d}_{\mathrm{S}} \boldsymbol{v}^{\mathrm{S}}}{\mathrm{d} t}=\nabla \cdot \mathrm{t}^{\mathrm{S}}+\boldsymbol{M}^{\mathrm{S}} \cdot \nabla \boldsymbol{B}^{\mathrm{S}}+\boldsymbol{P}^{\mathrm{S}}+\rho^{\mathrm{S}} \boldsymbol{f}^{\mathrm{S}} .
\end{aligned}
$$


(iii) Balances of energy:

$$
\begin{gathered}
\frac{\partial}{\partial t}\left(\rho^{\mathrm{F}} \epsilon^{\mathrm{F}}\right)+\nabla \cdot\left(\rho^{\mathrm{F}} \epsilon^{\mathrm{F}} \boldsymbol{v}^{\mathrm{F}}\right)=\mathrm{t}^{\mathrm{F}}: \nabla \boldsymbol{v}^{\mathrm{F}}-\boldsymbol{\nabla} \cdot \boldsymbol{q}^{\mathrm{F}}+\rho^{\mathrm{F}} r^{\mathrm{F}}+Q^{\mathrm{F}} \\
\frac{\partial}{\partial t}\left(\rho^{\mathrm{S}} \epsilon^{\mathrm{S}}\right)+\nabla \cdot\left(\rho^{\mathrm{S}} \epsilon^{\mathrm{S}} \boldsymbol{v}^{\mathrm{S}}\right)=\mathrm{t}^{\mathrm{S}}: \nabla \boldsymbol{v}^{\mathrm{S}}-\nabla \cdot \boldsymbol{q}^{\mathrm{S}}-\boldsymbol{M}^{\mathrm{S}} \cdot \frac{\mathrm{d}_{\mathrm{S}} \boldsymbol{B}^{\mathrm{S}}}{\mathrm{d} t}+\rho^{\mathrm{S}} r^{\mathrm{S}}+Q^{\mathrm{S}} .
\end{gathered}
$$

In the above equations, $\boldsymbol{P}^{\mathrm{F}}$ and $\boldsymbol{P}^{\mathrm{S}}$ are the momentum productions of the fluid and of the solid, $f^{\mathrm{F}}$ and $\boldsymbol{f}^{\mathrm{S}}$, the external non-magnetic body forces of the fluid and the solid, and $Q^{\mathrm{F}}$ and $Q^{\mathrm{S}}$, the energy productions of the fluid and of the solid. $\boldsymbol{M}^{\mathrm{S}}$ and $\boldsymbol{B}^{\mathrm{S}}$ represent the magnetization and magnetic flux of the solid, so that $\boldsymbol{M}^{\mathrm{S}} \cdot \boldsymbol{\nabla} \boldsymbol{B}^{\mathrm{S}}$ in equation (2.8) is the magnetic body force of the solid and $-\boldsymbol{M}^{\mathrm{S}} \cdot\left(\mathrm{d}_{\mathrm{S}} \boldsymbol{B}^{\mathrm{S}} / \mathrm{d} t\right)$ in equation (2.10) is the energy supply due to the magnetic field (Hutter \& van de Ven 1978; Eringen \& Maugin 1990). In equations (2.9) and (2.10), $\epsilon^{\mathrm{F}}$ and $\epsilon^{\mathrm{S}}$ denote the internal energy densities of the fluid and the solid, and $r^{\mathrm{F}}$ and $r^{\mathrm{S}}$ represent the external heat source of the fluid and solid. In order to ensure that the conservations of linear momentum and energy of the mixture as a whole hold true, the productions $\boldsymbol{P}^{\mathrm{F}}, \boldsymbol{P}^{\mathrm{S}}, Q^{\mathrm{F}}$ and $Q^{\mathrm{S}}$ shall be constrained by the following conditions (Bowen 1976; Müller 1985):

$$
\begin{gathered}
\boldsymbol{P}^{\mathrm{F}}+\boldsymbol{P}^{\mathrm{S}}=\mathbf{0}, \\
Q^{\mathrm{F}}+\boldsymbol{v}^{\mathrm{F}} \cdot \boldsymbol{P}^{\mathrm{F}}+Q^{\mathrm{S}}+\boldsymbol{v}^{\mathrm{S}} \cdot \boldsymbol{P}^{\mathrm{S}}=0 .
\end{gathered}
$$

With the specifications

$$
\begin{gathered}
\rho \epsilon=\rho^{\mathrm{F}} \epsilon^{\mathrm{F}}+\rho^{\mathrm{S}} \epsilon^{\mathrm{S}}, \quad \rho r=\rho^{\mathrm{F}} r^{\mathrm{F}}+\rho^{\mathrm{S}} r^{\mathrm{S}}, \\
\boldsymbol{q}=\boldsymbol{q}^{\mathrm{F}}+\boldsymbol{q}^{\mathrm{S}}+\rho^{\mathrm{F}} \epsilon^{\mathrm{F}} \overline{\boldsymbol{v}}^{\mathrm{F}}+\rho^{\mathrm{S}} \epsilon^{\mathrm{S}} \overline{\boldsymbol{v}}^{\mathrm{S}},
\end{gathered}
$$

and with the conditions (2.11) and (2.12), the energy balance equations of the fluid and the solid can be combined to be

$$
\frac{\partial}{\partial t}(\rho \epsilon)+\nabla \cdot(\rho \epsilon \boldsymbol{v})=\mathrm{t}^{\mathrm{F}}: \nabla \boldsymbol{v}^{\mathrm{F}}+\mathrm{t}^{\mathrm{S}}: \nabla \boldsymbol{v}^{\mathrm{S}}-\nabla \cdot \boldsymbol{q}-\boldsymbol{M}^{\mathrm{S}} \cdot \frac{\mathrm{d}_{\mathrm{S}} \boldsymbol{B}^{\mathrm{S}}}{\mathrm{d} t}+\rho r+\boldsymbol{P}^{\mathrm{S}} \cdot \boldsymbol{v}^{\mathrm{R}} .
$$

This is the macroscopic energy equation (3.6) used in the previous article (Chen $\&$ Yeh 2002a). It should be noted that no effect of spin $s$ is addressed in the above discussion. While the spin effect is included, an additional power, $c: \nabla\left(\Theta^{-1} \cdot s\right)$, contributed by couple stress $\boldsymbol{c}$ will be inserted in the energy equations (2.9), (2.10) and (2.14). Here, the symbol $\Theta$ represents the tensor of moment of inertia, which will be used in the following discussion.

\section{Distribution function of solid continuum}

In the mesoscopic theory of liquid crystals, the orientation of a molecule $\boldsymbol{n}^{\mathrm{LC}}$ is a mesoscopic variable to distinguish the different components of the mixture and hence all field quantities and field equations can be defined on the nematic space 
$\mathcal{R}_{x}^{3} \times \mathcal{S}^{2} \times \mathcal{R}_{t}$, where $\mathcal{S}^{2}$ stands for the unit sphere (Ehrentraut et al. 1997; Muschik et al. 2000, 2004; Papenfuss 2000). Two mesoscopic velocities associated with the liquid crystals can be defined as

$$
\boldsymbol{v} \equiv \frac{\mathrm{d}}{\mathrm{d} t} \boldsymbol{x}=\left.\frac{\partial \boldsymbol{x}}{\partial t}\right|_{X}, \quad \boldsymbol{u} \equiv \frac{\mathrm{d}}{\mathrm{d} t} \boldsymbol{n}^{\mathrm{LC}}=\left.\frac{\partial \boldsymbol{n}^{\mathrm{LC}}}{\partial t}\right|_{X},
$$

where $X$ and $\mathrm{d} / \mathrm{d} t$ indicate the material point and the material time derivative, and $\boldsymbol{v}$ and $\boldsymbol{u}$ are the material velocity and the orientation change velocity.

The concept of the mesoscopic variable $\boldsymbol{n}^{\mathrm{LC}}$ cannot be directly applied to the modelling of MR materials, in which the spherical particles are uniformly suspended in the non-magnetic fluid so that no particular geometric property of particles survives. However, in the presence of a magnetic field the particles will be magnetized and aggregate to form an orderly geometric structure. This phenomenon arises from the arrangement of particles rather than the intrinsic geometry of the particles as in liquid crystals. Yeh \& Chen (1997) and Chen \& Yeh $(2002 b)$ proposed a macroscopic internal variable with twofold average of microscopic distance among particles to model the behaviour of MR materials. Now in this paper we use a vector $l$ as a measure of orientation and distance among particles to characterize the aggregation of magnetized particles. The vector $\boldsymbol{l}$ is assigned to each particle and is a relative distance vector $\boldsymbol{r}_{\mathrm{AB}}$ between two nearest particles A and B. The length of $\boldsymbol{l}$ is the norm of $\boldsymbol{r}_{\mathrm{AB}}$, i.e. $\left|\boldsymbol{r}_{\mathrm{AB}}\right|$, and the orientation of $\boldsymbol{l}$ is defined such that the angle between $\boldsymbol{r}_{\mathrm{AB}}$ and the direction of an applied magnetic field $\boldsymbol{H}$ should not be greater than $90^{\circ}$. Since the constraint condition $\cos (\boldsymbol{l}, \boldsymbol{H}) \geq 0$ limits the range of $\boldsymbol{l}$ in the half space with coordinates $\left(l_{1}, l_{2}, l_{3}\right)$, for analytic convenience we enlarge the space of $\boldsymbol{l}$ and specify the symmetric condition as that used for the orientation $\boldsymbol{n}^{\mathrm{LC}}$ in liquid crystals. In this way the reverse direction, $-\boldsymbol{l}$, has the same significance as $\boldsymbol{l}$ and the distributions of $-\boldsymbol{l}$ and $\boldsymbol{l}$ are identical. Moreover, it should be noticed that the vector $\boldsymbol{l}$ will vanish when the applied magnetic field is turned off since the vector $l$ is a measure of the magnetic-induced structure which survives only in the presence of a magnetic field. Besides, the orientation of $\boldsymbol{l}$ depends on the direction of the applied magnetic field.

While comparing the vector $\boldsymbol{l}$ in MR materials with the vector $\boldsymbol{n}^{\mathrm{LC}}$ in liquid crystals, it should be emphasized that the vector $n^{\mathrm{LC}}$ is a true vector associated with the molecules, and its evolution in time is continuous, whereas the vector $\boldsymbol{l}$ is defined as a vector attached to the magnetized particles and its evolution in time might be discontinuous. Discontinuity occurs, for example, when another particle is also enlisted as the nearest neighbour to a considered particle since at this time the vector $\boldsymbol{l}$ becomes the average of both of the relative distance vectors. An illustration is given in figure 1 , where, in addition to $\mathrm{B}, \mathrm{C}$ becomes the particle that is of equal distance to A. So the vector $l$ becomes the average of both of the relative distance vectors. The property of the discontinuity of $\boldsymbol{l}$ resembles that of the discontinuity of the velocity $\boldsymbol{c}$ of a particle in the kinetic theory of gases when a collision occurs. Similar to the conservation of linear momentum before and after a collision, there is an analogous conservation while the discontinuity of $\boldsymbol{l}$ happens. Since the vector $\boldsymbol{l}$ is a mesoscopic variable that was used to distinguish the different components of the solid continuum mixture, the analogous conservation amounts to the conservation of the mass productions among these components of the solid mixture. However, it has been well known 


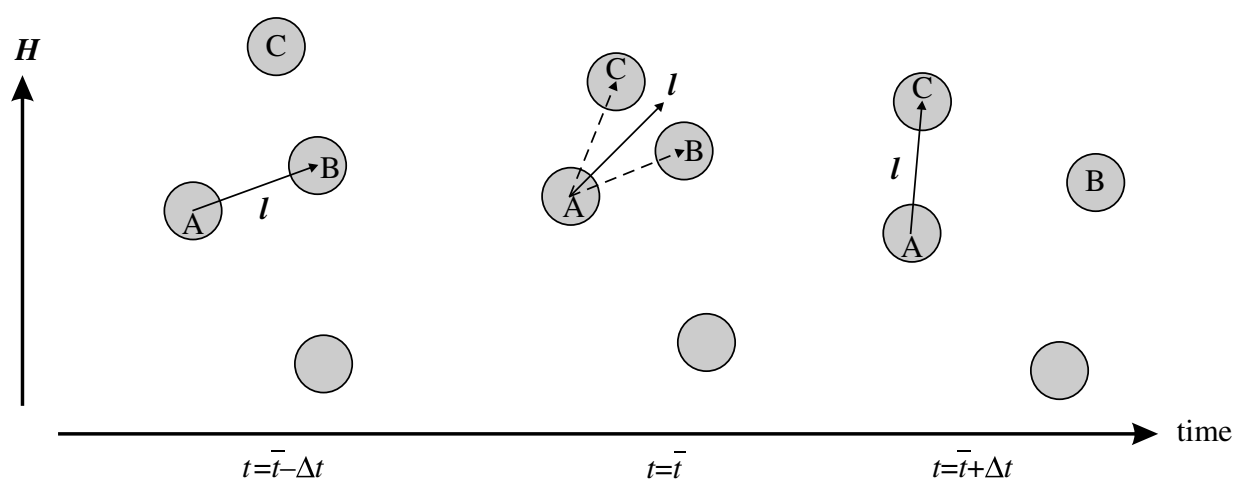

Figure 1. In the presence of the applied magnetic field $\boldsymbol{H}$, the vector $\boldsymbol{l}$ attached to the considered particle $\mathrm{A}$ is defined as the relative position vector from the particle A to its nearest particle $\mathrm{B}$. At time $t=\bar{t}$ the movement of the particles yields an equal distance between $\mathrm{A}$ and $\mathrm{C}$ to that between $\mathrm{A}$ and $\mathrm{B}$, which instantaneously makes the vector $\boldsymbol{l}$ become the average of the two relative position vectors. Then at time $t=\bar{t}+\Delta t$ a further change occurs when the particle $\mathrm{C}$ replaces $\mathrm{B}$ as the nearest particle to $\mathrm{A}$. The three configurations at $t=\bar{t}-\Delta t, t=\bar{t}$, and $t=\bar{t}+\Delta t$ manifest the timediscontinuity of the vector $l$.

that there is a production term in the mass balance equations in the theory of mixture with chemical reactions. Therefore, it is reasonable to treat the process of discontinuity of $\boldsymbol{l}$ as 'a pseudo chemical reaction' in the mesoscopic theory of MR materials.

Moreover, the vector $\boldsymbol{l}=\boldsymbol{l n}$ can be expressed by its length $l$ and its orientation $\boldsymbol{n}$, where $\boldsymbol{n}$ is a unit vector. The velocity $\dot{l}$ of the change in length and the orientation velocity $\dot{\boldsymbol{n}} \equiv \boldsymbol{u}$ are defined as

$$
\dot{l}=\lim _{\Delta t \rightarrow 0} \frac{l(t+\Delta t)-l(t)}{\Delta t}, \quad \boldsymbol{u}=\lim _{\Delta t \rightarrow 0} \frac{\boldsymbol{n}(t+\Delta t)-\boldsymbol{n}(t)}{\Delta t},
$$

so that the material time rate of change of $l$ is yielded by

$$
\boldsymbol{v}_{l}^{\mathrm{S}}=\frac{\mathrm{d}_{\mathrm{S}}}{\mathrm{d} t} \boldsymbol{l}=\boldsymbol{l} \boldsymbol{u}+\dot{i} \boldsymbol{n} .
$$

Since $\boldsymbol{n}$ is a unit vector, the orientation velocity $\boldsymbol{u}$ must be perpendicular to $\boldsymbol{n}$ and be a vector tangent to the surface of a unit sphere, i.e. $\boldsymbol{n} \cdot \boldsymbol{u}=0$. With the introduction of the unit vector $\boldsymbol{n}$, one can express the differential operator $\boldsymbol{\nabla}_{\boldsymbol{n}}$ by the azimuthal part of the spherical coordinates $(\theta, \phi)$,

$$
\nabla_{n}=s \frac{\partial}{\partial \theta}+\mathrm{t} \frac{1}{\sin \theta} \frac{\partial}{\partial \phi},
$$

where

$$
\begin{gathered}
\boldsymbol{s}=\cos \theta \cos \phi \boldsymbol{e}_{x}+\cos \theta \sin \phi \boldsymbol{e}_{y}-\sin \theta \boldsymbol{e}_{z}, \\
\mathrm{t}=-\sin \phi \boldsymbol{e}_{x}+\cos \phi \boldsymbol{e}_{y},
\end{gathered}
$$

with the unit base vectors $\left(\boldsymbol{e}_{\boldsymbol{x}}, \boldsymbol{e}_{\boldsymbol{y}}, \boldsymbol{e}_{\boldsymbol{z}}\right)$ in a Cartesian coordinate system. With the help of $\nabla_{n}$, we arrive at the definitions of the operators $\nabla_{l}$ and $\nabla_{l}^{2}$ as

$$
\nabla_{l}=\frac{1}{l} \nabla_{n}+n \frac{\partial}{\partial l}, \quad \nabla_{l}^{2}=\nabla_{l} \cdot \nabla_{l}=\frac{1}{l^{2}} \frac{\partial}{\partial l}\left(l^{2} \frac{\partial}{\partial l}\right)+\frac{1}{l^{2}} \nabla_{n}^{2}
$$


and some useful derivable identities

$$
\begin{aligned}
& \nabla_{l} l=\left(\frac{1}{l} \nabla_{n}+n \frac{\partial}{\partial l}\right)(\ln )=\boldsymbol{n n}+\mathrm{I}-\boldsymbol{n n}=\mathrm{I}, \\
& \nabla_{l} \cdot l=\left(\frac{1}{l} \nabla_{n}+n \frac{\partial}{\partial l}\right) \cdot(\ln )=\nabla_{n} \cdot \boldsymbol{n}+1=3 \\
& \nabla_{l}(\boldsymbol{l l})=\left(\frac{1}{l} \nabla_{n}+\boldsymbol{n} \frac{\partial}{\partial l}\right)(\boldsymbol{l l})=\mathbf{l} \boldsymbol{l}+\boldsymbol{n l n}+\boldsymbol{s} \boldsymbol{l} \boldsymbol{s}+\mathrm{t} \boldsymbol{l t} \mathbf{t}=\mathbf{l} \boldsymbol{l}+(\mathrm{Il})^{\mathrm{T}} \\
& \nabla_{l} \cdot(\boldsymbol{l l})=\left(\frac{1}{l} \nabla_{n}+\boldsymbol{n} \frac{\partial}{\partial l}\right) \cdot(\boldsymbol{l l})=\boldsymbol{n} \cdot \frac{\partial}{\partial l}\left(l^{2} \boldsymbol{n n}\right)+\frac{1}{l} \nabla_{n} \cdot\left(l^{2} \boldsymbol{n n}\right)=4 \boldsymbol{l} \\
& \nabla_{l}^{2}(\boldsymbol{l l})=2 \mathrm{l},
\end{aligned}
$$

which will be used in $\$ 6$. Here, $\mathrm{I}=\boldsymbol{n} \boldsymbol{n}+\boldsymbol{s} \boldsymbol{s}+\mathbf{t t}$ and the notation of tensor product $\boldsymbol{a} \boldsymbol{b}=\boldsymbol{a} \otimes \boldsymbol{b}$ for two arbitrary vectors $\boldsymbol{a}$ and $\boldsymbol{b}$ is used. The superscript ' $\mathrm{T}$ ' in equation (3.10) indicates the transpose of the last two indices, i.e.

$$
(\mathbf{l} \boldsymbol{l})^{\mathrm{T}}=[(\boldsymbol{n n}+\boldsymbol{s} \boldsymbol{s}+\mathrm{tt}) \boldsymbol{l}]^{\mathrm{T}}=(\boldsymbol{n} \boldsymbol{l n}+\boldsymbol{s l} \boldsymbol{s}+\mathrm{t} \boldsymbol{l t}) .
$$

For the solid continuum of MR materials we extend the six-dimensional nematic space $\mathcal{R}_{x}^{3} \times \mathcal{S}^{2} \times \mathcal{R}_{t}$ to the seven-dimensional mesoscopic space $\mathcal{R}_{x}^{3} \times \mathcal{M} \times \mathcal{R}_{t}$, where $\mathcal{M}$ is the manifold of the vector $\boldsymbol{l}$. The length of $\boldsymbol{l}$ is limited to the range $\left[l_{\min }, l_{\max }\right]$, where $l_{\min }$ is the diameter of the magnetized particle and $l_{\max }$ is constrained by the size of the materials. In the mesoscopic theory, the domain of field quantities is the set of the mesoscopic space $(\boldsymbol{x}, \boldsymbol{l}, t)$. The mesoscopic mass density in this seven-dimensional space is denoted by $\hat{\rho}^{\mathrm{S}}(\boldsymbol{x}, \boldsymbol{l}, t)$. The macroscopic mass density $\rho^{\mathrm{S}}(\boldsymbol{x}, t)$ then can be obtained by the integration of the mesoscopic mass density over the manifold $\mathcal{M}$,

$$
\rho^{\mathrm{S}}(\boldsymbol{x}, t)=\int_{l_{\min }}^{l_{\max }} \int_{S^{2}} \hat{\boldsymbol{\rho}}^{\mathrm{S}}(\boldsymbol{x}, \boldsymbol{l}, t) l^{2} \mathrm{~d} \boldsymbol{n} \mathrm{d} l=\int_{\mathcal{M}} \hat{\boldsymbol{\rho}}^{\mathrm{S}}(\boldsymbol{x}, \boldsymbol{l}, t) \mathrm{d} v_{\mathcal{M}}
$$

The mesoscopic distribution function, which is the probability density to find a solid particle in space-time $(\boldsymbol{x}, t)$ with the variable $\boldsymbol{l}$, can be defined as the mesoscopic mass density divided by the macroscopic mass density (Blenk \& Muschik 1991),

$$
\hat{f}(\boldsymbol{x}, \boldsymbol{l}, t)=\frac{\hat{\rho}^{\mathrm{S}}(\boldsymbol{x}, \boldsymbol{l}, t)}{\rho^{\mathrm{S}}(\boldsymbol{x}, t)} .
$$

From equation (3.14) there follows the normalization condition,

$$
\int_{\mathcal{M}} \hat{f}(\boldsymbol{x}, \boldsymbol{l}, t) \mathrm{d} v_{\mathcal{M}}=1 .
$$

As soon as the distribution function $\hat{f}$ has been defined, we can further give the macroscopic solid material velocity $\boldsymbol{v}^{\mathrm{S}}(\boldsymbol{x}, t)$ as the average of the mesoscopic 
solid material velocity $\hat{\boldsymbol{v}}^{\mathrm{S}}(\bullet)$ over the manifold $\mathcal{M}$ :

$$
\begin{aligned}
& \boldsymbol{v}^{\mathrm{S}}(\boldsymbol{x}, t)=\int_{\mathcal{M}} \hat{f}(\bullet) \hat{\boldsymbol{v}}^{\mathrm{S}}(\bullet) \mathrm{d} v_{\mathcal{M}}, \quad \text { or } \\
& \rho^{\mathrm{S}}(\boldsymbol{x}, t) \boldsymbol{v}^{\mathrm{S}}(\boldsymbol{x}, t)=\int_{\mathcal{M}} \hat{\rho}^{\mathrm{S}}(\bullet) \hat{\boldsymbol{v}}^{\mathrm{S}}(\bullet) \mathrm{d} v_{\mathcal{M}},
\end{aligned}
$$

where the abbreviation $(\bullet)=(\boldsymbol{x}, \boldsymbol{l}, t)$ has been used. To distinguish the symbols used in the macroscopic and mesoscopic space, e.g. $\rho^{\mathrm{S}}(\boldsymbol{x}, t)$ and $\hat{\rho}^{\mathrm{S}}(\bullet)$, the quantities with ^ indicate the associated functions used in the mesoscopic space. By means of the corresponding mesoscopic averaging similar to or the same as equation (3.16), we will introduce other macroscopic quantities in later sections.

\section{Mesoscopic balance equations for the solid particles}

In this modelling of MR fluids, the solid continuum is a mixture of different components with indices $\boldsymbol{l}$. Under the assumption that the fluid continuum is spinless and shall be considered macroscopically, there is no fluid component at the meso-continuum. In this mesoscopic approach the general mesoscopic balance equations of the solid continuum read (Blenk et al. 1991a)

$$
\frac{\mathrm{d}_{\mathrm{S}}}{\mathrm{d} t} \int_{\Omega} \hat{\Phi}(\bullet) \mathrm{d} v \mathrm{~d} v_{\mathcal{M}}=\int_{\Omega}\left(-\nabla \cdot \hat{\Phi}^{\phi x}(\bullet)-\nabla_{l} \cdot \hat{\Phi}^{\phi l}(\bullet)+\hat{\Phi}^{\pi}(\bullet)+\hat{\Phi}^{\varsigma}(\bullet)\right) \mathrm{d} v \mathrm{~d} v_{\mathcal{M}},
$$

where $\Omega$ represents a region in $\mathcal{R}_{x}^{3} \times \mathcal{R}_{t} \times \mathcal{M} . \hat{\Phi}^{\phi x}(\bullet)$ and $\hat{\Phi}^{\phi l}(\bullet)$ are the nonconvective fluxes through the surface in the configuration space $\mathcal{R}_{x}^{3}$ and on the manifold $\mathcal{M}$. $\hat{\Phi}^{\pi}(\bullet)$ and $\hat{\Phi}^{\varsigma}(\bullet)$ denote the production and the supply of $\hat{\Phi}(\bullet)$. The total time derivative $\left(\mathrm{d}_{\mathrm{S}} / \mathrm{d} t\right)$ in the first term of equation (4.1) can be moved inside the integral by use of the generalized Reynolds transport theorem (Blenk \& Muschik 1991),

$$
\frac{\mathrm{d}_{\mathrm{S}}}{\mathrm{d} t} \int_{\Omega} \hat{\Phi}(\bullet) \mathrm{d} v \mathrm{~d} v_{\mathcal{M}}=\int_{\Omega}\left[\frac{\partial}{\partial t} \hat{\Phi}(\bullet)+\nabla \cdot\left(\hat{\boldsymbol{v}}^{\mathrm{S}}(\bullet) \hat{\Phi}(\bullet)\right)+\nabla_{l} \cdot\left(\hat{\boldsymbol{v}}_{l}^{\mathrm{S}}(\bullet) \hat{\Phi}(\bullet)\right)\right] \mathrm{d} v \mathrm{~d} v_{\mathcal{M}}
$$

Supposing the field quantities are sufficiently smooth, we have the general expression of mesoscopic balance equations in local form,

$$
\frac{\partial}{\partial t} \hat{\Phi}(\bullet)+\nabla \cdot\left(\hat{\boldsymbol{v}}^{\mathrm{S}}(\bullet) \hat{\Phi}(\bullet)+\hat{\Phi}^{\phi x}(\bullet)\right)+\nabla_{l} \cdot\left(\hat{\boldsymbol{v}}_{l}^{\mathrm{S}}(\bullet) \hat{\Phi}(\bullet)+\hat{\Phi}^{\phi l}(\bullet)\right)=\hat{\Phi}^{\pi}(\bullet)+\hat{\Phi}^{\varsigma}(\bullet) .
$$

For the mesoscopic balance of mass, we have

$$
\begin{gathered}
\hat{\Phi} \equiv \hat{\rho}^{\mathrm{S}}(\bullet), \quad \hat{\Phi}^{\phi x} \equiv \mathbf{0}, \quad \hat{\Phi}^{\phi l} \equiv \mathbf{0}, \quad \hat{\Phi}^{\pi} \equiv \hat{\tau}(\bullet), \quad \hat{\Phi}^{\varsigma} \equiv 0, \\
\frac{\partial}{\partial t} \hat{\rho}^{\mathrm{S}}(\bullet)+\nabla \cdot\left(\hat{\rho}^{\mathrm{S}}(\bullet) \hat{\boldsymbol{v}}^{S}(\bullet)\right)+\nabla_{l} \cdot\left(\hat{\rho}^{S}(\bullet) \hat{\boldsymbol{v}}_{l}^{\mathrm{S}}(\bullet)\right)=\hat{\tau}(\bullet),
\end{gathered}
$$


where $\hat{\tau}(\bullet)$ represents the mass production which arises from the mechanism that other components $\boldsymbol{l}^{\prime}$ instantaneously change to the component $\boldsymbol{l}$ at time $t$. In other words, it comes from the contribution of the time-discontinuity of $\boldsymbol{l}$ as has been shown in figure 1 . Notice that the analogous term of mass production appears in the theory of mixture when the effect of chemical reaction is taken into account. Since the conservation of total mass of the solid medium holds true, the constraint condition,

$$
\int_{\mathcal{M}} \hat{\tau}(\bullet) \mathrm{d} v_{\mathcal{M}}=0
$$

must be obeyed.

Moreover, for the mesoscopic balance of linear momentum, we have

$$
\begin{gathered}
\hat{\Phi} \equiv \hat{\boldsymbol{\rho}}^{\mathrm{S}}(\bullet) \hat{\boldsymbol{v}}^{\mathrm{S}}(\bullet), \quad \hat{\Phi}^{\phi x} \equiv-\hat{\mathrm{t}}^{\mathrm{S}}(\bullet), \quad \hat{\Phi}^{\phi l} \equiv-\hat{\mathrm{t}}_{l}^{\mathrm{S}}(\bullet), \\
\hat{\Phi}^{\pi} \equiv \hat{\boldsymbol{m}}^{l}(\bullet)+\hat{\boldsymbol{\tau}}(\bullet) \hat{\boldsymbol{v}}^{S}(\bullet)+\hat{\boldsymbol{P}}^{\mathrm{S}}(\bullet) \\
\hat{\boldsymbol{\Phi}}^{\mathrm{S}} \equiv \hat{\boldsymbol{M}}^{\mathrm{S}}(\bullet) \cdot \nabla \boldsymbol{B}^{\mathrm{S}}(\boldsymbol{x}, t)+\hat{\boldsymbol{\rho}}^{\mathrm{S}}(\bullet) \hat{\boldsymbol{f}}^{\mathrm{S}}(\bullet), \\
\frac{\partial}{\partial t}\left(\hat{\boldsymbol{\rho}}^{\mathrm{S}}(\bullet) \hat{\boldsymbol{v}}^{\mathrm{S}}(\bullet)\right)+\nabla \cdot\left(\hat{\boldsymbol{\rho}}^{\mathrm{S}}(\bullet) \hat{\boldsymbol{v}}^{\mathrm{S}}(\bullet) \hat{\boldsymbol{v}}^{\mathrm{S}}(\bullet)-\hat{\mathrm{t}}^{\mathrm{S}}(\bullet)\right)+\nabla_{\boldsymbol{l}} \cdot\left(\hat{\boldsymbol{\rho}}^{\mathrm{S}}(\bullet) \hat{\boldsymbol{v}}_{\boldsymbol{l}}^{\mathrm{S}}(\bullet) \hat{\boldsymbol{v}}^{\mathrm{S}}(\bullet)-\hat{\mathrm{t}}_{l}^{\mathrm{S}}(\bullet)\right) \\
=\hat{\boldsymbol{M}}^{\mathrm{S}}(\bullet) \cdot \nabla \boldsymbol{B}^{\mathrm{S}}(\boldsymbol{x}, t)+\hat{\boldsymbol{\rho}}^{\mathrm{S}}(\bullet) \hat{\boldsymbol{f}}^{\mathrm{S}}(\bullet)+\hat{\boldsymbol{m}}^{l}(\bullet)+\hat{\boldsymbol{\tau}}(\bullet) \hat{\boldsymbol{v}}^{\mathrm{S}}(\bullet)+\hat{\boldsymbol{P}}^{\mathrm{S}}(\bullet),
\end{gathered}
$$

where $\hat{\mathrm{t}}^{\mathrm{S}}(\bullet)$ and $\hat{\mathrm{t}}_{l}^{\mathrm{S}}(\bullet)$ are, respectively, the solid mesoscopic stress tensor on the configuration space and the solid momentum flux on the manifold $\mathcal{M}$. The supply is composed of the contribution from the magnetic effect $\hat{\boldsymbol{M}}^{\mathrm{S}}(\bullet) \cdot \nabla \boldsymbol{B}^{\mathrm{S}}(\boldsymbol{x}, t)$ and from the non-magnetic force $\hat{\rho}^{\mathrm{S}}(\bullet) \hat{\boldsymbol{f}}^{\mathrm{S}}(\bullet)$. The production is the combination of the three momentum productions $\hat{\boldsymbol{m}}^{l}(\bullet), \hat{\boldsymbol{\tau}}(\bullet) \hat{\boldsymbol{v}}^{\mathrm{S}}(\bullet)$, and $\hat{\boldsymbol{P}}^{\mathrm{S}}(\bullet)$. The first production $\hat{\boldsymbol{m}}^{l}(\bullet)$ comes from other components of the solid mixture to the solid component with mesoscopic variable $\boldsymbol{l}$, the second one $\hat{\boldsymbol{\tau}}(\bullet) \boldsymbol{v}^{\mathrm{S}}(\bullet)$ is due to the mass production, and the last one $\hat{\boldsymbol{P}}^{\mathrm{S}}(\bullet)$ is from the carrying fluid to the solid component with mesoscopic variable $\boldsymbol{l}$. Different from the conservation of mass for the solid continuum, the conservation of linear momentum for the solid continuum cannot be held since there exists an exchange of linear momentum between the solid and the fluid continua.

Third, with the specifications

$$
\begin{aligned}
& \hat{\boldsymbol{\Phi}} \equiv \hat{\rho}^{\mathrm{S}}(\bullet) \hat{\boldsymbol{S}}(\bullet)=\hat{\rho}^{\mathrm{S}}(\bullet)\left(\boldsymbol{x} \times \hat{\boldsymbol{v}}^{\mathrm{S}}(\bullet)+\hat{\boldsymbol{s}}^{\mathrm{S}}(\bullet)\right), \\
& \hat{\Phi}^{\phi x} \equiv-\left(\boldsymbol{x} \times\left(\hat{\mathrm{t}}^{\mathrm{S}}(\bullet)\right)^{\mathrm{T}}\right)^{\mathrm{T}}-\hat{\boldsymbol{c}}^{\mathrm{S}}(\bullet), \\
& \hat{\Phi}^{\phi l} \equiv-\left(\boldsymbol{x} \times\left(\hat{\mathrm{t}}_{l}^{\mathrm{S}}(\bullet)\right)^{\mathrm{T}}\right)^{\mathrm{T}}-\hat{\boldsymbol{c}}_{l}^{\mathrm{S}}(\bullet), \\
& \hat{\Phi}^{\pi} \equiv \boldsymbol{x} \times\left(\hat{\boldsymbol{m}}^{l}(\bullet)+\hat{\tau}(\bullet) \hat{\boldsymbol{v}}^{\mathrm{S}}(\bullet)+\hat{\boldsymbol{P}}^{\mathrm{S}}(\bullet)\right)+\hat{\boldsymbol{m}}^{\mathrm{S}}(\bullet), \\
& \hat{\Phi}^{\varsigma} \equiv \boldsymbol{x} \times\left(\hat{\boldsymbol{M}}^{\mathrm{S}}(\bullet) \cdot \nabla \boldsymbol{B}^{\mathrm{S}}(\boldsymbol{x}, t)\right)+\hat{\rho}^{\mathrm{S}}(\bullet) \boldsymbol{x} \times \hat{\boldsymbol{f}}^{\mathrm{S}}(\bullet)+\hat{\boldsymbol{M}}^{\mathrm{S}}(\bullet) \times \boldsymbol{B}^{\mathrm{S}}(\boldsymbol{x}, t),
\end{aligned}
$$


the mesoscopic balance of angular momentum may be written in the form

$$
\begin{aligned}
\frac{\partial}{\partial t}\left(\hat{\boldsymbol{\rho}}^{\mathrm{S}}(\bullet) \hat{\boldsymbol{S}}(\bullet)\right)+\nabla \cdot\left(\hat{\boldsymbol{\rho}}^{\mathrm{S}}(\bullet) \hat{\boldsymbol{v}}^{\mathrm{S}}(\bullet) \hat{\boldsymbol{S}}(\bullet)-\left(\boldsymbol{x} \times\left(\hat{\mathrm{t}}^{\mathrm{S}}(\bullet)\right)^{\mathrm{T}}\right)^{\mathrm{T}}-\hat{\boldsymbol{c}}^{\mathrm{S}}(\bullet)\right) \\
\quad+\nabla_{l} \cdot\left(\hat{\boldsymbol{\rho}}^{\mathrm{S}}(\bullet) \hat{\boldsymbol{v}}_{l}^{\mathrm{S}}(\bullet) \hat{\boldsymbol{S}}(\bullet)-\left(\boldsymbol{x} \times\left(\hat{\mathrm{t}}_{l}^{\mathrm{S}}(\bullet)\right)^{\mathrm{T}}\right)^{\mathrm{T}}-\hat{\boldsymbol{c}}_{l}^{\mathrm{S}}(\bullet)\right) \\
=\boldsymbol{x} \times\left(\hat{\boldsymbol{M}}^{\mathrm{S}}(\bullet) \cdot \nabla \boldsymbol{B}^{\mathrm{S}}(\boldsymbol{x}, t)\right)+\hat{\boldsymbol{\rho}}^{\mathrm{S}}(\bullet) \boldsymbol{x} \times \hat{\boldsymbol{f}}^{\mathrm{S}}(\bullet)+\hat{\boldsymbol{M}}^{\mathrm{S}}(\bullet) \times \boldsymbol{B}^{\mathrm{S}}(\boldsymbol{x}, t) \\
\quad+\boldsymbol{x} \times\left(\hat{\boldsymbol{m}}^{l}(\bullet)+\hat{\tau}(\bullet) \hat{\boldsymbol{v}}^{\mathrm{S}}(\bullet)+\hat{\boldsymbol{P}}^{\mathrm{S}}(\bullet)\right)+\hat{\boldsymbol{m}}^{\mathrm{S}}(\bullet),
\end{aligned}
$$

where $\hat{\boldsymbol{s}}^{\mathrm{S}}(\bullet), \hat{\boldsymbol{c}}^{\mathrm{S}}(\bullet)$, and $\hat{\boldsymbol{c}}_{l}^{\mathrm{S}}(\bullet)$ are, respectively, the spin density, the mesoscopic couple stress tensor, and the angular momentum flux of the solid on the manifold $\mathcal{M} . \hat{\boldsymbol{S}}(\bullet)$ is the total angular momentum. The production of spin from other components of the solid continuum is assumed to be $\hat{\boldsymbol{m}}^{\mathrm{s}}(\bullet)$, which is constrained by the condition

$$
\int_{\mathcal{M}} \hat{\boldsymbol{m}}^{\mathrm{s}}(\bullet) \mathrm{d} v_{\mathcal{M}}=\mathbf{0}
$$

This condition originates from the assumption that the carrying fluid is spinless and then the production of spin from the fluid to the solid is zero. By introducing the balance equation of linear momentum into the balance equation of angular momentum, the balance equation of spin can be expressed as

$$
\begin{gathered}
\frac{\partial}{\partial t}\left(\hat{\rho}^{\mathrm{S}}(\bullet) \hat{\boldsymbol{s}}^{\mathrm{S}}(\bullet)\right)+\nabla \cdot\left(\hat{\boldsymbol{\rho}}^{\mathrm{S}}(\bullet) \hat{\boldsymbol{v}}^{\mathrm{S}}(\bullet) \hat{\boldsymbol{s}}^{\mathrm{S}}(\bullet)-\hat{\boldsymbol{c}}^{\mathrm{S}}(\bullet)\right)+\nabla_{l} \cdot\left(\hat{\boldsymbol{\rho}}^{\mathrm{S}}(\bullet) \hat{\boldsymbol{v}}_{l}^{\mathrm{S}}(\bullet) \hat{\boldsymbol{s}}^{\mathrm{S}}(\bullet)-\hat{\boldsymbol{c}}_{l}^{\mathrm{S}}(\bullet)\right) \\
\quad=\epsilon: \hat{\mathrm{t}}^{\mathrm{S}}(\bullet)+\hat{\boldsymbol{M}}^{\mathrm{S}}(\bullet) \times \boldsymbol{B}^{\mathrm{S}}(\boldsymbol{x}, t)+\hat{\boldsymbol{m}}^{\mathrm{S}}(\bullet)
\end{gathered}
$$

where the index form for $\epsilon: \hat{\mathrm{t}}^{\mathrm{S}}$ is $\epsilon_{i j k} \hat{t}_{i j}^{\mathrm{S}}$ with $\epsilon$ the permutation symbol.

Fourth, if we consider

$$
\begin{gathered}
\hat{\Phi} \equiv \hat{\boldsymbol{\rho}}^{\mathrm{S}}(\bullet) \hat{e}^{\mathrm{S}}(\bullet)=\hat{\boldsymbol{\rho}}^{\mathrm{S}}(\bullet)\left(\frac{1}{2} \hat{\boldsymbol{v}}^{\mathrm{S}}(\bullet) \cdot \hat{\boldsymbol{v}}^{\mathrm{S}}(\bullet)+\frac{1}{2} \hat{\boldsymbol{s}}^{\mathrm{S}}(\bullet) \cdot \Theta^{-1} \cdot \hat{\boldsymbol{s}}^{\mathrm{S}}(\bullet)+\hat{\varepsilon}^{\mathrm{S}}(\bullet)\right) \\
\hat{\Phi}^{\phi x} \equiv-\hat{\mathrm{t}}^{\mathrm{S}}(\bullet) \cdot \hat{\boldsymbol{v}}^{\mathrm{S}}(\bullet)-\hat{\boldsymbol{c}}^{\mathrm{S}}(\bullet) \cdot \Theta^{-1} \cdot \hat{\boldsymbol{s}}^{\mathrm{S}}(\bullet)+\hat{\boldsymbol{q}}^{\mathrm{S}}(\bullet) \\
\hat{\Phi}^{\phi l} \equiv-\hat{\mathrm{t}}_{l}^{\mathrm{S}}(\bullet) \cdot \hat{\boldsymbol{v}}^{\mathrm{S}}(\bullet)-\hat{\boldsymbol{c}}_{l}^{\mathrm{S}}(\bullet) \cdot \Theta^{-1} \cdot \hat{\boldsymbol{s}}^{\mathrm{S}}(\bullet)+\hat{\boldsymbol{q}}_{l}^{\mathrm{S}}(\bullet) \\
\hat{\Phi}^{\pi} \equiv\left(\hat{\boldsymbol{m}}^{l}(\bullet)+\hat{\boldsymbol{P}}^{\mathrm{S}}(\bullet)\right) \cdot \hat{\boldsymbol{v}}^{\mathrm{S}}(\bullet)+\hat{\boldsymbol{m}}^{\mathrm{S}}(\bullet) \cdot \Theta^{-1} \cdot \hat{\boldsymbol{s}}^{\mathrm{S}}(\bullet)+Q^{\mathrm{S}}(\bullet) \\
+\hat{\tau}(\bullet)\left(\frac{1}{2} \hat{\boldsymbol{v}}^{\mathrm{S}}(\bullet) \cdot \hat{\boldsymbol{v}}^{\mathrm{S}}(\bullet)+\frac{1}{2} \hat{\boldsymbol{s}}^{\mathrm{S}}(\bullet) \cdot \Theta^{-1} \cdot \hat{\boldsymbol{s}}^{\mathrm{S}}(\bullet)\right) \\
\hat{\Phi}^{\varsigma} \equiv \hat{\boldsymbol{\rho}}^{\mathrm{S}}(\bullet) \hat{r}^{\mathrm{S}}(\bullet)+\left(\left(\hat{\boldsymbol{M}}^{\mathrm{S}}(\bullet) \cdot \nabla\right) \boldsymbol{B}^{\mathrm{S}}(\boldsymbol{x}, t)+\hat{\boldsymbol{\rho}}^{\mathrm{S}}(\bullet) \hat{\boldsymbol{f}}^{\mathrm{S}}(\bullet)\right) \cdot \hat{\boldsymbol{v}}^{\mathrm{S}}(\bullet) \\
+\left(\boldsymbol{\epsilon}: \hat{\mathrm{t}}^{\mathrm{S}}(\bullet)+\hat{\boldsymbol{M}}^{\mathrm{S}}(\bullet) \times \boldsymbol{B}^{\mathrm{S}}(\boldsymbol{x}, t)\right) \cdot \Theta^{-1} \cdot \hat{\boldsymbol{s}}^{\mathrm{S}}(\bullet)-\hat{\boldsymbol{M}}^{\mathrm{S}}(\bullet) \cdot \frac{\mathrm{d}_{\mathrm{S}}}{\mathrm{d} t} \boldsymbol{B}^{\mathrm{S}}(\boldsymbol{x}, t)
\end{gathered}
$$


for the mesoscopic balance equation of total energy of the solid in MR fluids, then we have

$$
\begin{aligned}
& \frac{\partial}{\partial t}\left(\hat{\rho}^{\mathrm{S}}(\bullet) \hat{e}^{\mathrm{S}}(\bullet)\right)+\nabla \cdot\left(\hat{\boldsymbol{\rho}}^{\mathrm{S}}(\bullet) \hat{\boldsymbol{v}}^{\mathrm{S}}(\bullet) \hat{e}^{\mathrm{S}}(\bullet)-\hat{\mathrm{t}}^{\mathrm{S}}(\bullet) \cdot \hat{\boldsymbol{v}}^{\mathrm{S}}(\bullet)-\hat{\boldsymbol{c}}^{\mathrm{S}}(\bullet) \cdot \Theta^{-1} \cdot \hat{\boldsymbol{s}}^{\mathrm{S}}(\bullet)+\hat{\boldsymbol{q}}^{\mathrm{S}}(\bullet)\right) \\
& \quad+\nabla_{l} \cdot\left(\hat{\boldsymbol{\rho}}^{\mathrm{S}}(\bullet) \hat{\boldsymbol{v}}_{l}^{\mathrm{S}}(\bullet) \hat{e}^{\mathrm{S}}(\bullet)-\hat{\mathrm{t}}_{l}^{\mathrm{S}}(\bullet) \cdot \hat{\boldsymbol{v}}^{\mathrm{S}}(\bullet)-\hat{\boldsymbol{c}}_{l}^{\mathrm{S}}(\bullet) \cdot \Theta^{-1} \cdot \hat{\boldsymbol{s}}^{\mathrm{S}}(\bullet)+\hat{\boldsymbol{q}}_{l}^{\mathrm{S}}(\bullet)\right) \\
& =\hat{\rho}^{\mathrm{S}}(\bullet) \hat{r}^{\mathrm{S}}(\bullet)+\left(\left(\hat{\boldsymbol{M}}^{\mathrm{S}}(\bullet) \cdot \nabla\right) \boldsymbol{B}^{\mathrm{S}}(\boldsymbol{x}, t)+\hat{\boldsymbol{\rho}}^{\mathrm{S}}(\bullet) \hat{\boldsymbol{f}}^{\mathrm{S}}(\bullet)+\hat{\boldsymbol{m}}^{l}(\bullet)+\hat{\boldsymbol{P}}^{\mathrm{S}}(\bullet)\right) \cdot \hat{\boldsymbol{v}}^{\mathrm{S}}(\bullet) \\
& \quad+\left(\epsilon: \mathrm{t}^{\mathrm{S}}(\bullet)+\hat{\boldsymbol{M}}^{\mathrm{S}}(\bullet) \times \boldsymbol{B}^{\mathrm{S}}(\boldsymbol{x}, t)+\hat{\boldsymbol{m}}^{\mathrm{S}}(\bullet)\right) \cdot \Theta^{-1} \cdot \hat{\boldsymbol{s}}^{\mathrm{S}}(\bullet) \\
& \quad-\hat{\boldsymbol{M}}^{\mathrm{S}}(\bullet) \cdot \frac{\mathrm{d}_{\mathrm{S}} \mathrm{d} t}{\mathrm{~d} t} \boldsymbol{B}^{\mathrm{S}}(\boldsymbol{x}, t)+\hat{Q}^{\mathrm{S}}(\bullet)+\hat{\tau}(\bullet)\left(\frac{1}{2} \hat{\boldsymbol{v}}^{\mathrm{S}}(\bullet) \cdot \hat{\boldsymbol{v}}^{\mathrm{S}}(\bullet)+\frac{1}{2} \hat{\boldsymbol{s}}^{\mathrm{S}}(\bullet) \cdot \Theta^{-1} \cdot \hat{\boldsymbol{s}}^{\mathrm{S}}(\bullet)\right),
\end{aligned}
$$

where $\hat{\rho}^{\mathrm{S}} \hat{r}^{\mathrm{S}}, \hat{\boldsymbol{q}}^{\mathrm{S}}$, and $\hat{\boldsymbol{q}}_{l}^{\mathrm{S}}$ are the non-magnetic solid supply, the mesoscopic solid energy flux on the configuration space, and that on the manifold $\mathcal{M}$, respectively. $\Theta$ is the tensor of moment of inertia. The total energy density of the solid continuum $\hat{e}^{\mathrm{S}}(\bullet)$ is composed of kinetic energy, rotational energy and internal energy $\hat{\varepsilon}^{\mathrm{S}}(\bullet)$. The energy production $\hat{Q}^{\mathrm{S}}(\bullet)$ is included to account for the energy exchange from the fluid and other components of the solid mixture to the solid component with mesoscopic variable $\boldsymbol{l}$. With the help of balance equations of mass, linear momentum, and spin, the balance of total energy can be further simplified to the balance of internal energy as

$$
\begin{aligned}
& \frac{\partial}{\partial t}\left(\hat{\rho}^{\mathrm{S}}(\bullet) \hat{\varepsilon}^{\mathrm{S}}(\bullet)\right)+\nabla \cdot\left(\hat{\boldsymbol{\rho}}^{\mathrm{S}}(\bullet) \hat{\boldsymbol{v}}^{\mathrm{S}}(\bullet) \hat{\varepsilon}^{\mathrm{S}}(\bullet)+\hat{\boldsymbol{q}}^{\mathrm{S}}(\bullet)\right)+\nabla_{l} \cdot\left(\hat{\boldsymbol{\rho}}^{\mathrm{S}}(\bullet) \hat{\boldsymbol{v}}_{l}^{\mathrm{S}}(\bullet) \hat{\varepsilon}^{\mathrm{S}}(\bullet)+\hat{\boldsymbol{q}}_{l}^{\mathrm{S}}(\bullet)\right) \\
& =\hat{\boldsymbol{\rho}}^{\mathrm{S}}(\bullet) \hat{r}^{\mathrm{S}}(\bullet)-\hat{\boldsymbol{M}}^{\mathrm{S}}(\bullet) \cdot \frac{\mathrm{d}_{\mathrm{S}}}{\mathrm{d} t} \boldsymbol{B}^{\mathrm{S}}(\boldsymbol{x}, t)+\left(\hat{\mathrm{t}}^{\mathrm{S}}(\bullet): \nabla+\hat{\mathrm{t}}_{l}^{\mathrm{S}}(\bullet): \nabla_{l}\right) \hat{\boldsymbol{v}}^{\mathrm{S}}(\bullet) \\
& \quad+\left(\hat{\boldsymbol{c}}^{\mathrm{S}}(\bullet): \nabla+\hat{\boldsymbol{c}}_{l}^{\mathrm{S}}(\bullet): \nabla_{l}\right)\left(\Theta^{-1} \cdot \hat{\boldsymbol{s}}^{\mathrm{S}}(\bullet)\right)+\hat{Q}^{\mathrm{S}}(\bullet) .
\end{aligned}
$$

In addition, the balance of entropy is represented as the second law of thermodynamics, which demands that the entropy of an isolated system never be decreased. Since this law only seems to be valid in the macroscopic scale, no mesoscopic balance equations of entropy are required.

\section{Macroscopic field quantities and balance equations}

In the literature, there exist a lot of research works on bridging the gap between two different scales of space. The theory of electrodynamics is the first successful paradigm to correlate the macroscopic EM fields with their microscopic counterparts by the spatial average with respect to a continuous and smooth test function, whose scale is large compared to the atomic dimension (de Groot \& Suttorp 1972; Jackson 1975). However, it should be noted that not all the EM quantities can be achieved in this way. For example, polarization and magnetization, which have their significance only on the macroscopic scale, are brought out in the process of average of charge and current over space. Another paradigm is the theory of extended thermodynamics, in which the macroscopic 
hydrodynamic theory and the microscopic kinetic theory of gases are linked (Müller \& Ruggeri 1998). Still another paradigm is the study of micromechanics or statistical theory of granular mechanics, in which the microscopic forces among particles are associated with the macroscopic stress tensor (Edwards \& Grinev 2001).

The macroscopic physical quantities of a mixture are the macroscopic averages of individual quantities, and the macroscopic physical quantities in the mesoscopic theory are the mesoscopic averages over the mesoscopic manifold. For the macroscopic-mesoscopic mixture of MR fluids we combine both averages to obtain the macroscopic quantities.

Now we consider the balance equation of mass. In view of equations (3.13) and (3.16), we take the first step to integrate the mesoscopic balance of mass for the solid continuum (4.5) over the manifold $\mathcal{M}$ and then have

$$
\frac{\partial}{\partial t} \rho^{\mathrm{S}}(\boldsymbol{x}, t)+\nabla \cdot\left(\rho^{\mathrm{S}}(\boldsymbol{x}, t) \boldsymbol{v}^{\mathrm{S}}(\boldsymbol{x}, t)\right)=0,
$$

which is the same expression as the second equation in (2.7) if the condition

$$
\int_{\mathcal{M}} \nabla_{l}\left(\hat{\rho}^{\mathrm{S}}(\bullet) \hat{\boldsymbol{v}}_{l}^{\mathrm{S}}(\bullet)\right) \mathrm{d} v_{\mathcal{M}}=0
$$

is satisfied. This condition can be extended to a more general case, in which

$$
\int_{\mathcal{M}} \nabla_{l}\left(\hat{\boldsymbol{v}}_{\boldsymbol{l}}^{\mathrm{S}}(\bullet) \hat{g}_{1}(\bullet)+\hat{\boldsymbol{g}}_{2}(\bullet)\right) \mathrm{d} v_{\mathcal{M}}=0
$$

holds true for any continuous function $\hat{g}_{1}$ and the flux $\hat{\boldsymbol{g}}_{2}$. Condition (5.3) can be easily proved by considering $\boldsymbol{n} \cdot \boldsymbol{u}=0$ and the fact that the boundary of $\mathcal{M}$ is null, $\partial \mathcal{M}=0$, giving no rise of a flux on $\partial \mathcal{M}$. The second step is to add the mass balance of the fluid and solid continua in equation (2.7) to yield the macroscopic balance equation of mass,

$$
\frac{\partial}{\partial t} \rho(\boldsymbol{x}, t)+\nabla \cdot(\rho(\boldsymbol{x}, t) \boldsymbol{v}(\boldsymbol{x}, t))=0,
$$

where

$$
\begin{array}{r}
\rho(\boldsymbol{x}, t)=\rho^{\mathrm{F}}(\boldsymbol{x}, t)+\rho^{\mathrm{S}}(\boldsymbol{x}, t)=\rho^{\mathrm{F}}(\boldsymbol{x}, t)+\int_{\mathcal{M}} \hat{\rho}^{\mathrm{S}}(\bullet) \mathrm{d} v_{\mathcal{M}}, \\
\rho(\boldsymbol{x}, t) \boldsymbol{v}(\boldsymbol{x}, t)=\rho^{\mathrm{F}}(\boldsymbol{x}, t) \boldsymbol{v}^{\mathrm{F}}(\boldsymbol{x}, t)+\rho^{\mathrm{S}}(\boldsymbol{x}, t) \boldsymbol{v}^{\mathrm{S}}(\boldsymbol{x}, t) \\
=\rho^{\mathrm{F}}(\boldsymbol{x}, t) \boldsymbol{v}^{\mathrm{F}}(\boldsymbol{x}, t)+\int_{\mathcal{M}} \hat{\rho}^{\mathrm{S}}(\bullet) \hat{\boldsymbol{v}}^{\mathrm{S}}(\bullet) \mathrm{d} v_{\mathcal{M}} .
\end{array}
$$

Furthermore, for handling the balance of linear momentum we integrate the mesoscopic balance of linear momentum (4.10) over the manifold $\mathcal{M}$ and then arrive at

$$
\begin{gathered}
\frac{\partial}{\partial t}\left(\rho^{\mathrm{S}}(\boldsymbol{x}, t) \boldsymbol{v}^{\mathrm{S}}(\boldsymbol{x}, t)\right)+\nabla \cdot\left(\rho^{\mathrm{S}}(\boldsymbol{x}, t) \boldsymbol{v}^{\mathrm{S}}(\boldsymbol{x}, t) \boldsymbol{v}^{\mathrm{S}}(\boldsymbol{x}, t)-\mathrm{t}^{\mathrm{S}}(\boldsymbol{x}, t)\right) \\
\quad=\boldsymbol{M}^{\mathrm{S}}(\boldsymbol{x}, t) \cdot \boldsymbol{\nabla} \boldsymbol{B}^{\mathrm{S}}(\boldsymbol{x}, t)+\boldsymbol{P}^{\mathrm{S}}(\boldsymbol{x}, t)+\rho^{\mathrm{S}}(\boldsymbol{x}, t) \boldsymbol{f}^{\mathrm{S}}(\boldsymbol{x}, t),
\end{gathered}
$$


which is the same as the second equation in (2.8) if we require condition (5.3) and set

$$
\begin{gathered}
\mathrm{t}^{\mathrm{S}}(\boldsymbol{x}, t)=\int_{\mathcal{M}}\left[\hat{\mathrm{t}}^{\mathrm{S}}(\bullet)-\hat{\boldsymbol{\rho}}^{\mathrm{S}}(\bullet)\left(\hat{\boldsymbol{v}}^{\mathrm{S}}(\bullet)-\boldsymbol{v}^{\mathrm{S}}(\boldsymbol{x}, t)\right)\left(\hat{\boldsymbol{v}}^{\mathrm{S}}(\bullet)-\boldsymbol{v}^{\mathrm{S}}(\boldsymbol{x}, t)\right)\right] \mathrm{d} v_{\mathcal{M}}, \\
\boldsymbol{M}^{\mathrm{S}}(\boldsymbol{x}, t)=\int_{\mathcal{M}} \hat{\boldsymbol{M}}^{\mathrm{S}}(\bullet) \mathrm{d} v_{\mathcal{M}} \\
\boldsymbol{f}^{\mathrm{S}}(\boldsymbol{x}, t)=\int_{\mathcal{M}} \hat{f}(\bullet) \hat{\boldsymbol{f}}^{\mathrm{S}}(\bullet) \mathrm{d} v_{\mathcal{M}}, \\
\boldsymbol{P}^{\mathrm{S}}(\boldsymbol{x}, t)=\int_{\mathcal{M}}\left(\hat{\boldsymbol{m}}^{l}(\bullet)+\hat{\boldsymbol{\tau}}(\bullet) \hat{\boldsymbol{v}}^{\mathrm{S}}(\bullet)+\boldsymbol{P}^{\mathrm{S}}(\bullet)\right) \mathrm{d} v_{\mathcal{M}},
\end{gathered}
$$

where $\boldsymbol{P}^{\mathrm{S}}(\boldsymbol{x}, t)$ is the momentum production of the solid by the fluid continuum at the point $(\boldsymbol{x}, t)$. The combination of the linear momentum balance equations for the fluid and solid continua in equation (2.8) will give rise to

$$
\begin{aligned}
& \frac{\partial}{\partial t}(\rho(\boldsymbol{x}, t) \boldsymbol{v}(\boldsymbol{x}, t))+\nabla \cdot(\rho(\boldsymbol{x}, t) \boldsymbol{v}(\boldsymbol{x}, t) \boldsymbol{v}(\boldsymbol{x}, t)-\mathrm{t}(\boldsymbol{x}, t)) \\
& \quad=\boldsymbol{M}^{\mathrm{S}}(\boldsymbol{x}, t) \cdot \nabla \boldsymbol{B}^{\mathrm{S}}(\boldsymbol{x}, t)+\rho(\boldsymbol{x}, t) \boldsymbol{f}(\boldsymbol{x}, t),
\end{aligned}
$$

where

$$
\begin{aligned}
\mathrm{t}(\boldsymbol{x}, t)= & \mathrm{t}^{\mathrm{F}}(\boldsymbol{x}, t)+\mathrm{t}^{\mathrm{S}}(\boldsymbol{x}, t)-\left(\rho^{\mathrm{F}} \overline{\boldsymbol{v}}^{\mathrm{F}} \overline{\boldsymbol{v}}^{\mathrm{F}}+\rho^{\mathrm{S}} \overline{\boldsymbol{v}}^{\mathrm{S}} \overline{\boldsymbol{v}}^{\mathrm{S}}\right) \\
= & \mathrm{t}^{\mathrm{F}}(\boldsymbol{x}, t)-\left(\rho^{\mathrm{F}} \overline{\boldsymbol{v}}^{\mathrm{F}} \overline{\boldsymbol{v}}^{\mathrm{F}}+\rho^{\mathrm{S}} \overline{\boldsymbol{v}}^{\mathrm{S}} \overline{\boldsymbol{v}}^{\mathrm{S}}\right)+\int_{\mathcal{M}}\left[\hat{\mathrm{t}}^{\mathrm{S}}(\bullet)-\hat{\rho}^{\mathrm{S}}(\bullet)\left(\hat{\boldsymbol{v}}^{\mathrm{S}}(\bullet)\right.\right. \\
& \left.\left.-\boldsymbol{v}^{\mathrm{S}}(\boldsymbol{x}, t)\right)\left(\hat{\boldsymbol{v}}^{\mathrm{S}}(\bullet)-\boldsymbol{v}^{\mathrm{S}}(\boldsymbol{x}, t)\right)\right] \mathrm{d} v_{\mathcal{M}}, \\
& \rho(\boldsymbol{x}, t) \boldsymbol{f}(\boldsymbol{x}, t)=\rho^{\mathrm{F}}(\boldsymbol{x}, t) \boldsymbol{f}^{\mathrm{F}}(\boldsymbol{x}, t)+\int_{\mathcal{M}} \hat{\rho}^{\mathrm{S}}(\bullet) \hat{\boldsymbol{f}}^{\mathrm{S}}(\bullet) \mathrm{d} v_{\mathcal{M}} .
\end{aligned}
$$

As for the macroscopic balance of angular momentum, one usually considers the balance of spin instead of the balance of total angular momentum. By the mesoscopic average of (4.18) over the manifold $\mathcal{M}$, the macroscopic balance of spin for the solid continuum of MR materials can be written as

$$
\begin{aligned}
& \frac{\partial}{\partial t}\left(\rho^{\mathrm{S}}(\boldsymbol{x}, t) \boldsymbol{s}^{\mathrm{S}}(\boldsymbol{x}, t)\right)+\nabla \cdot\left(\rho^{\mathrm{S}}(\boldsymbol{x}, t) \boldsymbol{v}^{\mathrm{S}}(\boldsymbol{x}, t) \boldsymbol{s}^{\mathrm{S}}(\boldsymbol{x}, t)-\boldsymbol{c}^{\mathrm{S}}(\boldsymbol{x}, t)\right) \\
& =\epsilon: \mathrm{t}^{\mathrm{S}}(\boldsymbol{x}, t)+\boldsymbol{M}^{\mathrm{S}}(\boldsymbol{x}, t) \times \boldsymbol{B}^{\mathrm{S}}(\boldsymbol{x}, t),
\end{aligned}
$$

with the following identifications:

$$
\begin{gathered}
\rho^{\mathrm{S}}(\boldsymbol{x}, t) \boldsymbol{s}^{\mathrm{S}}(\boldsymbol{x}, t)=\int_{\mathcal{M}} \hat{\boldsymbol{\rho}}^{\mathrm{S}}(\bullet) \hat{\boldsymbol{s}}^{\mathrm{S}}(\bullet) \mathrm{d} v_{\mathcal{M}}, \\
\boldsymbol{c}^{\mathrm{S}}(\boldsymbol{x}, t)=\int_{\mathcal{M}}\left[\hat{\boldsymbol{c}}^{\mathrm{S}}(\bullet)-\hat{\rho}^{\mathrm{S}}(\bullet)\left(\hat{\boldsymbol{v}}^{\mathrm{S}}(\bullet)-\boldsymbol{v}^{\mathrm{S}}(\boldsymbol{x}, t)\right) \hat{\boldsymbol{s}}^{\mathrm{S}}(\bullet)\right] \mathrm{d} v_{\mathcal{M}}
\end{gathered}
$$


Since we have assumed that the fluid continuum is spinless, the macroscopic balance of spin for MR materials becomes

$$
\begin{aligned}
& \frac{\partial}{\partial t}(\rho(\boldsymbol{x}, t) \boldsymbol{s}(\boldsymbol{x}, t))+\nabla \cdot(\rho(\boldsymbol{x}, t) \boldsymbol{v}(\boldsymbol{x}, t) \boldsymbol{s}(\boldsymbol{x}, t)-\boldsymbol{c}(\boldsymbol{x}, t)) \\
& \quad=\epsilon: \mathrm{t}^{\mathrm{S}}(\boldsymbol{x}, t)+\boldsymbol{M}^{\mathrm{S}}(\boldsymbol{x}, t) \times \boldsymbol{B}^{\mathrm{S}}(\boldsymbol{x}, t),
\end{aligned}
$$

where

$$
\begin{gathered}
\rho(\boldsymbol{x}, t) \boldsymbol{s}(\boldsymbol{x}, t)=\rho^{\mathrm{S}}(\boldsymbol{x}, t) \boldsymbol{s}^{\mathrm{S}}(\boldsymbol{x}, t)=\int_{\mathcal{M}} \hat{\rho}^{\mathrm{S}}(\bullet) \hat{s}^{\mathrm{S}}(\bullet) \mathrm{d} v_{\mathcal{M}} \\
\boldsymbol{c}(\boldsymbol{x}, t)=-\rho^{\mathrm{S}} \overline{\boldsymbol{v}}^{\mathrm{S}} \boldsymbol{s}^{\mathrm{S}}+\int_{\mathcal{M}}\left[\hat{\boldsymbol{c}}^{\mathrm{S}}(\bullet)-\hat{\boldsymbol{\rho}}^{\mathrm{S}}(\bullet)\left(\hat{\boldsymbol{v}}^{\mathrm{S}}(\bullet)-\boldsymbol{v}^{\mathrm{S}}(\boldsymbol{x}, t)\right) \hat{\boldsymbol{s}}^{\mathrm{S}}(\bullet)\right] \mathrm{d} v_{\mathcal{M}}
\end{gathered}
$$

The macroscopic balance equation of internal energy for the solid continuum of MR materials can be obtained by integration of the corresponding mesoscopic equation (4.25) over the manifold $\mathcal{M}$ as

$$
\begin{aligned}
\frac{\partial}{\partial t}\left(\rho^{\mathrm{S}}(\boldsymbol{x}, t) \varepsilon^{\mathrm{S}}(\boldsymbol{x}, t)\right)+\nabla \cdot\left(\rho^{\mathrm{S}}(\boldsymbol{x}, t) \boldsymbol{v}^{\mathrm{S}}(\boldsymbol{x}, t) \varepsilon^{\mathrm{S}}(\boldsymbol{x}, t)+\boldsymbol{q}^{\mathrm{S}}(\boldsymbol{x}, t)\right) \\
=\rho^{\mathrm{S}}(\boldsymbol{x}, t) r^{\mathrm{S}}(\boldsymbol{x}, t)-\boldsymbol{M}^{\mathrm{S}}(\boldsymbol{x}, t) \cdot \frac{\mathrm{d}_{\mathrm{S}} \boldsymbol{B}^{\mathrm{S}}}{\mathrm{d} t}(\boldsymbol{x}, t)+\mathrm{t}^{\mathrm{S}}(\boldsymbol{x}, t): \nabla \boldsymbol{v}^{\mathrm{S}}(\boldsymbol{x}, t) \\
\quad+\boldsymbol{c}^{\mathrm{S}}(\boldsymbol{x}, t): \nabla\left(\Theta^{-1} \cdot \boldsymbol{s}^{\mathrm{S}}(\boldsymbol{x}, t)\right)+Q^{\mathrm{S}}(\boldsymbol{x}, t),
\end{aligned}
$$

with the specifications of the relations of the macroscopic and mesoscopic quantities:

$$
\begin{aligned}
& \rho^{\mathrm{S}}(\boldsymbol{x}, t) \varepsilon^{\mathrm{S}}(\boldsymbol{x}, t)=\int_{\mathcal{M}} \hat{\boldsymbol{\rho}}^{\mathrm{S}}(\bullet)\left[\hat{\varepsilon}^{\mathrm{S}}(\bullet)+\frac{1}{2} \delta \hat{\boldsymbol{v}}^{\mathrm{S}}(\bullet) \cdot \delta \hat{\boldsymbol{v}}^{\mathrm{S}}(\bullet)\right. \\
& \left.+\frac{1}{2} \delta \hat{\boldsymbol{s}}^{\mathrm{S}}(\bullet) \cdot \Theta^{-1} \cdot \hat{\delta} s^{\mathrm{S}}(\bullet)\right] \mathrm{d} v_{\mathcal{M}}, \\
& \boldsymbol{q}^{\mathrm{S}}(\boldsymbol{x}, t)=\int_{\mathcal{M}}\left[\hat{\boldsymbol{q}}^{\mathrm{S}}(\bullet)+\left(\hat{\boldsymbol{\rho}}^{\mathrm{S}}(\bullet) \hat{\varepsilon}^{\mathrm{S}}(\bullet)+\frac{1}{2} \hat{\boldsymbol{\rho}}^{\mathrm{S}}(\bullet) \delta \hat{\boldsymbol{v}}^{\mathrm{S}}(\bullet) \cdot \delta \hat{\boldsymbol{v}}^{\mathrm{S}}(\bullet)\right.\right. \\
& \left.-\left(\hat{\mathrm{t}}^{\mathrm{S}}(\bullet)\right)^{\mathrm{T}}\right) \cdot \delta \hat{\boldsymbol{v}}^{\mathrm{S}}(\bullet)+\left({ }_{2} \hat{\rho}^{\mathrm{S}}(\bullet) \delta \hat{\boldsymbol{s}}^{\mathrm{S}}(\bullet) \cdot \Theta^{-1} \cdot \delta \hat{\boldsymbol{s}}^{\mathrm{S}}(\bullet)\right) \delta \hat{\boldsymbol{v}}^{\mathrm{S}}(\bullet) \\
& \left.-\left(\hat{\boldsymbol{c}}^{\mathrm{S}}(\bullet)\right)^{\mathrm{T}} \cdot \Theta^{-1} \cdot \delta \hat{\boldsymbol{s}}^{\mathrm{S}}(\bullet)\right] \mathrm{d} v_{\mathcal{M}}, \\
& \rho^{\mathrm{S}}(\boldsymbol{x}, t) r^{\mathrm{S}}(\boldsymbol{x}, t)=\int_{\mathcal{M}}\left[\hat{\rho}^{\mathrm{S}}(\bullet) \hat{r}^{\mathrm{S}}(\bullet)+\left(\hat{\boldsymbol{M}}^{\mathrm{S}}(\bullet) \cdot \nabla \boldsymbol{B}^{\mathrm{S}}(\boldsymbol{x}, t)\right.\right. \\
& \left.+\hat{\boldsymbol{\rho}}^{\mathrm{S}}(\bullet) \hat{\boldsymbol{f}}^{\mathrm{S}}(\bullet)\right) \cdot \delta \hat{\boldsymbol{v}}^{\mathrm{S}}(\bullet)+\left(\hat{\boldsymbol{M}}^{\mathrm{S}}(\bullet) \times \boldsymbol{B}^{\mathrm{S}}(\boldsymbol{x}, t)\right. \\
& \left.\left.+\epsilon: \hat{\mathrm{t}}^{\mathrm{S}}(\bullet)\right) \cdot \Theta^{-1} \cdot \delta \hat{\boldsymbol{s}}^{\mathrm{S}}(\bullet)\right] \mathrm{d} v_{\mathcal{M}}, \\
& Q^{\mathrm{S}}(\boldsymbol{x}, t)=-\boldsymbol{P}^{\mathrm{S}}(\boldsymbol{x}, t) \cdot \boldsymbol{v}^{\mathrm{S}}(\boldsymbol{x}, t)+\int_{\mathcal{M}}\left[\hat{Q}^{\mathrm{S}}(\bullet)+\left(\hat{\boldsymbol{m}}^{l}(\bullet)+\hat{\boldsymbol{P}}^{\mathrm{S}}(\bullet)\right) \cdot \hat{\boldsymbol{v}}^{\mathrm{S}}(\bullet)\right. \\
& +\hat{\boldsymbol{m}}^{\mathrm{S}}(\bullet) \cdot \Theta^{-1} \cdot \hat{\boldsymbol{s}}^{\mathrm{S}}(\bullet)+\hat{\tau}(\bullet)\left(\frac{1}{2} \hat{\boldsymbol{v}}^{\mathrm{S}}(\bullet) \cdot \hat{\boldsymbol{v}}^{\mathrm{S}}(\bullet)\right. \\
& \left.\left.+{ }_{2} \frac{1}{\boldsymbol{s}^{\mathrm{S}}}(\bullet) \cdot \Theta^{-1} \cdot \hat{\boldsymbol{s}}^{\mathrm{S}}(\bullet)\right)\right] \mathrm{d} v_{\mathcal{M}},
\end{aligned}
$$


where $\delta$ indicates deviation from the mean value,

$$
\delta \hat{\boldsymbol{v}}^{\mathrm{S}}(\bullet) \equiv \hat{\boldsymbol{v}}^{\mathrm{S}}(\bullet)-\boldsymbol{v}^{\mathrm{S}}(\boldsymbol{x}, t), \quad \delta \hat{\boldsymbol{s}}^{\mathrm{S}}(\bullet) \equiv \hat{\boldsymbol{s}}^{\mathrm{S}}(\bullet)-\boldsymbol{s}^{\mathrm{S}}(\boldsymbol{x}, t) .
$$

Then, by the same manipulation for solid-fluid mixture as in $\S 2$, the energy equation for the solid continuum in equation (5.21) can be combined with that for the fluid continuum, (2.9), to obtain the macroscopic energy equation, (2.14), for the MR materials. For those details we refer to $\S 2$. Note that the energy equations for the solid, fluid, and the whole mixture will be used if one engages in the discussion of constitutive modelling and the physics related to energy change, such as thermal conduction and a variational approach.

From the above derivation, it becomes clear that the macroscopic quantities are derived from the mesoscopic average of their corresponding mesoscopic quantities plus other related mesoscopic quantities. We have also achieved a clear picture of the finer composition of the various macroscopic balance equations for the macroscopic-mesoscopic MR mixture.

For the whole system, especially for the mesoscopic system, to be complete, we should both study the role played by the mesoscopic distribution function and consider the constitutive equations characterizing the response of materials. In this work, we primarily focus on the discussion of the mesoscopic distribution function. The latter issue, that is a complete development of constitutive modelling, is not treated here for the following reason. From a theoretical viewpoint, one requires the knowledge of energy function and dissipative mechanism of the system when the constitutive modelling in the mesoscopic space is considered. In addition to the theoretical consideration such as the restriction of the second law of thermodynamics and the principle of material frame indifference, more experimental works are required for the development. In the literature, Blenk et al. (1992) have derived the macroscopic constitutive equations for liquid crystals induced by their mesoscopic orientation distribution. Papenfuss \& Muschik (1995) have considered the constitutive relations for twodimensional liquid crystals. Up to now, even though several researchers have devoted effects to the discussion of mesoscopic constitutive relations, a complete mesoscopic constitutive theory has still not been well developed (Muschik et al. 2004). The major problem lies in the fact that the constitutive modelling involves not only macroscopic variables, but also mesoscopic variables in the higherdimensional mesoscopic space. For MR materials, the set of constitutive variables could be specified by $\left(\boldsymbol{l}, \hat{\rho}^{\mathrm{S}}(\bullet), \nabla \hat{\rho}^{\mathrm{S}}(\bullet), \nabla_{l} \hat{\rho}^{\mathrm{S}}(\bullet), \rho^{\mathrm{F}}(\boldsymbol{x}, t), \theta(\boldsymbol{x}, t), \boldsymbol{B}^{\mathrm{S}}(\boldsymbol{x}, t)\right)$ or other alternatives. It is obvious that the diversity of the set definitely gives rise to a much more complicated analysis, which deserves a forthcoming paper to deal with in a systematic way and is thus beyond the scope of the current paper.

\section{Second moment equations for the mesoscopic distribution function}

The mesoscopic distribution $\hat{f}(\bullet)$ in equation (3.14) embraces all the information of the arrangement of solid particles in MR materials. This is similar to the fact that in liquid crystals the orientation distribution function contains the information of the orientation of microscopic molecules. It is also similar to the fact that in the kinetic theory of gases the phase density $f_{\mathrm{B}}(\boldsymbol{x}, \boldsymbol{c}, t)$, which is the number density of atoms at point $\boldsymbol{x}$ and time $t$ with velocity $\boldsymbol{c}$, provides the 
microscopic basis of macroscopic rational extended thermodynamics (Truesdell 1984; Müller \& Ruggeri 1998; Liu 2002). The phase density in the kinetic theory of gases obeys the Boltzmann equation,

$$
\frac{\partial f_{\mathrm{B}}}{\partial t}+\boldsymbol{c} \cdot \nabla f_{\mathrm{B}}+\overline{\boldsymbol{F}} \cdot \nabla_{c} f_{\mathrm{B}}=\int\left(f_{\mathrm{B}}^{\prime} f_{\mathrm{B}}^{1 \prime}-f_{\mathrm{B}} f_{\mathrm{B}}^{1}\right) r g \sin \theta \mathrm{d} \theta \mathrm{d} \epsilon \mathrm{d} \boldsymbol{c}^{1},
$$

where $\overline{\boldsymbol{F}}$ stands for the sum of the specific external body force and inertial acceleration of a particle, and the right-hand side of equation (6.1) comes from the contribution of collisions. By means of the phase density most macroscopic thermodynamic quantities can be obtained by taking the moments with respect to the velocity $\boldsymbol{c}$ or the peculiar velocity $\boldsymbol{C}=\boldsymbol{c}-\boldsymbol{v}$ as

$$
\boldsymbol{F}_{i_{1} i_{2} \cdots i_{N}}=\int m \boldsymbol{c}_{i_{1}} \boldsymbol{c}_{i_{2}} \cdots \boldsymbol{c}_{i_{N}} f_{\mathrm{B}} \mathrm{d} \boldsymbol{c}, \quad \text { or } \quad \boldsymbol{G}_{i_{1} i_{2} \cdots i_{N}}=\int m \boldsymbol{C}_{i_{1}} \boldsymbol{C}_{i_{2}} \cdots \boldsymbol{C}_{i_{N}} f_{\mathrm{B}} \mathrm{d} \boldsymbol{c} .
$$

In particular the mass density $\rho$, linear momentum density $\rho v_{i}$, internal energy density $\rho \epsilon$, and heat flux $q_{i}$ are related to the different orders of moments by

$$
\rho=F, \quad \rho v_{i}=F_{i}, \quad \rho \epsilon=\frac{1}{2} G_{i i}, \quad q_{i}=\frac{1}{2} G_{i j j} .
$$

The multiplication of the Boltzmann equation by $m \boldsymbol{c}_{i_{1}} \boldsymbol{c}_{i_{2}} \cdots \boldsymbol{c}_{i_{N}}$ and then integration over all $\boldsymbol{c}$ leads to

$$
\frac{\partial F_{i_{1} i_{2} \cdots i_{N}}}{\partial t}+\frac{\partial F_{i_{1} i_{2} \cdots i_{N j}}}{\partial x_{j}}-N F_{i_{1} i_{2} \cdots i_{N-1}} \bar{F}_{i_{N}}-2 N F_{i_{1} i_{2} \cdots i_{N-1}} W_{i_{N} k}=S_{i_{1} i_{2} \cdots i_{N}},
$$

where $S_{i_{1} i_{2} \cdots i_{N}}$ represents the collision production and $W_{i j}$ is the matrix of angular velocity of the frame with respect to an inertial frame. In equation (6.4) the zero and first-order moment equations read

$$
\frac{\partial \rho}{\partial t}+\nabla \cdot(\rho \boldsymbol{v})=0, \quad \text { and } \quad \frac{\partial \rho \boldsymbol{v}}{\partial t}+\nabla \cdot \boldsymbol{F}_{i_{1} i_{2}}^{\mathrm{T}}-\rho \overline{\boldsymbol{F}}-2 \rho \boldsymbol{W} \cdot \boldsymbol{v}=0,
$$

which represent the balance equations of mass and linear momentum.

The above approach can analogously be applied to the mesoscopic theory of the solid continuum of MR materials, if the role of the phase density is replaced by the mesoscopic distribution function $\hat{f}(\bullet)$. Analogous to that the phase density satisfies the Boltzmann equation, then by use of the definition of $\hat{f}(\bullet)$ in equation (3.14), the distribution function is governed by the equation (Blenk et al. 1991a)

$$
\begin{aligned}
& \frac{\partial \hat{f}(\bullet)}{\partial t}+\nabla \cdot\left(\hat{f}(\bullet) \hat{\boldsymbol{v}}^{\mathrm{S}}(\bullet)\right)+\nabla_{l} \cdot\left(\hat{f}(\bullet) \hat{\boldsymbol{v}}_{l}^{\mathrm{S}}(\bullet)\right)+\hat{f}(\bullet)\left[\frac{\partial}{\partial t}+\hat{\boldsymbol{v}}^{\mathrm{S}}(\bullet) \cdot \nabla\right] \ln \rho^{\mathrm{S}}(\boldsymbol{x}, t) \\
& \quad=\frac{\hat{\tau}(\bullet)}{\rho^{\mathrm{S}}(\boldsymbol{x}, t)} \equiv \hat{\bar{\tau}}(\bullet),
\end{aligned}
$$

or else

$$
\begin{aligned}
& \frac{\partial \hat{f}(\bullet)}{\partial t}+\boldsymbol{v}^{\mathrm{S}}(\boldsymbol{x}, t) \cdot \nabla \hat{f}(\bullet)+\nabla_{l} \cdot\left(\hat{f}(\bullet) \hat{\boldsymbol{v}}_{l}^{\mathrm{S}}(\bullet)\right) \\
& \quad+\nabla \cdot\left(\delta \hat{\boldsymbol{v}}^{\mathrm{S}}(\bullet) \hat{f}(\bullet)\right)+\hat{f}(\bullet) \delta \hat{\boldsymbol{v}}^{\mathrm{S}}(\bullet) \cdot \nabla \ln \rho^{\mathrm{S}}(\boldsymbol{x}, t)=\hat{\bar{\tau}}(\bullet)
\end{aligned}
$$


which is exactly the mesoscopic balance equation of mass (4.5) in terms of the distribution function $\hat{f}(\bullet)$. $\hat{\bar{\tau}}(\bullet)$, appearing on the right-hand side of equation (6.6) or (6.7), represents the production from the pseudo-chemical reaction. Then one can follow a similar procedure used in extended thermodynamics, which has been mentioned in equation (6.2), to associate the distribution function with the related macroscopic quantities by taking the moments with respect to the vector $\boldsymbol{l}$ over the manifold $\boldsymbol{M}$ as

$$
\mathrm{A}_{\alpha_{1} \alpha_{2} \cdots \alpha_{m}}(\boldsymbol{x}, t) \equiv \int_{\mathcal{M}} \hat{f}(\bullet) \boldsymbol{l}_{\alpha_{1}} \boldsymbol{l}_{\alpha_{2}} \cdots \boldsymbol{l}_{\alpha_{m}} \mathrm{~d} v_{\mathcal{M}},
$$

where $\mathrm{A}_{\alpha_{1} \alpha_{2} \cdots \alpha_{m}}$ is a tensor of order $m$. Since the distribution function $\hat{f}(\bullet)$ is endowed with the symmetry condition

$$
\hat{f}(\boldsymbol{x}, \boldsymbol{l}, t)=\hat{f}(\boldsymbol{x},-\boldsymbol{l}, t),
$$

the tensors of odd orders must vanish. Then, multiplication of (6.7) by $\boldsymbol{l}_{\alpha_{1}} \boldsymbol{l}_{\alpha_{2}} \cdots \boldsymbol{l}_{\alpha_{m}}$ and integration over the manifold $\mathcal{M}$ will lead to the general evolution equation for the macroscopic internal variable $\mathbf{A}_{\alpha_{1} \alpha_{2} \cdots \alpha_{m}}(\boldsymbol{x}, t)$ as (Muschik et al. 2000, 2004)

$$
\begin{aligned}
& \frac{\mathrm{d}_{\mathrm{S}}}{\mathrm{d} t} \mathrm{~A}_{\alpha_{1} \alpha_{2} \cdots \alpha_{m}}(\boldsymbol{x}, t)+\int_{\mathcal{M}} \boldsymbol{l}_{\alpha_{1}} \boldsymbol{l}_{\alpha_{2}} \cdots \boldsymbol{l}_{\alpha_{m}} \nabla_{\boldsymbol{l}} \cdot\left(\hat{f}(\bullet) \hat{\boldsymbol{v}}_{\boldsymbol{l}}^{\mathrm{S}}(\bullet)\right) \mathrm{d} v_{\mathcal{M}}+\nabla \cdot \int_{\mathcal{M}} \delta \hat{\boldsymbol{v}}^{\mathrm{S}}(\bullet) \boldsymbol{l}_{\alpha_{1}} \boldsymbol{l}_{\alpha_{2}} \\
& \cdots \boldsymbol{l}_{\alpha_{m}} \hat{f}(\bullet) \mathrm{d} v_{\mathcal{M}}+\int_{\mathcal{M}} \boldsymbol{l}_{\alpha_{1}} \boldsymbol{l}_{\alpha_{2}} \cdots \boldsymbol{l}_{\alpha_{m}} \hat{f}(\bullet) \delta \hat{\boldsymbol{v}}^{\mathrm{S}}(\bullet) \cdot \nabla \ln \rho^{\mathrm{S}}(\boldsymbol{x}, t) \mathrm{d} v_{\mathcal{M}} \\
& \quad=\int_{\mathcal{M}} \boldsymbol{l}_{\alpha_{1}} \boldsymbol{l}_{\alpha_{2}} \cdots \boldsymbol{l}_{\alpha_{m}} \hat{\bar{\tau}}(\bullet) \mathrm{d} v_{\mathcal{M}},
\end{aligned}
$$

where equation (6.8) has been used. Equation (6.9) shows that the macroscopic evolution equation for $\boldsymbol{A}_{\alpha_{1} \alpha_{2} \cdots \alpha_{m}}(\boldsymbol{x}, t)$ is derived from the mesoscopic distribution function. In order to get a more simplified form of equation (6.9), we require a further assumption and more constitutive information on the mesoscopic background such as explicit expressions for the functions $\hat{\boldsymbol{v}}^{\mathrm{S}}(\bullet), \hat{\boldsymbol{v}}_{\mathbf{l}}^{\mathrm{S}}(\bullet)$, and $\hat{\overline{\boldsymbol{\tau}}}(\bullet)$.

For simplicity we concentrate on the discussion of the second moment of the distribution function and from equation (6.9) we have

$$
\begin{aligned}
& \frac{\mathrm{d}_{\mathrm{S}}}{\mathrm{d} t} \mathrm{~A}_{2}(\boldsymbol{x}, t)+\int_{\mathcal{M}} \boldsymbol{l l} \nabla_{\boldsymbol{l}} \cdot\left(\hat{f}(\bullet) \hat{\boldsymbol{v}}_{\boldsymbol{l}}^{\mathrm{S}}(\bullet)\right) \mathrm{d} v_{\mathcal{M}} \\
& \quad+\nabla \cdot \int_{\mathcal{M}} \delta \hat{\boldsymbol{v}}^{\mathrm{S}}(\bullet) \boldsymbol{l l} f(\bullet) \mathrm{d} v_{\mathcal{M}}+\int_{\mathcal{M}} \boldsymbol{l l} \hat{f}(\bullet) \delta \hat{\boldsymbol{v}}^{\mathrm{S}}(\bullet) \cdot \nabla \ln \rho^{\mathrm{S}}(\boldsymbol{x}, t) \mathrm{d} v_{\mathcal{M}}=\int_{\mathcal{M}} \boldsymbol{l l} \hat{\bar{\tau}}(\bullet) \mathrm{d} v
\end{aligned}
$$

where $\mathrm{A}_{2}(\boldsymbol{x}, t)=\int \hat{f}(\bullet) \boldsymbol{l} \mathrm{d} v_{\mathcal{M}}$. The reduced macroscopic form of equation $(6.10)$ can be achieved only if the mesoscopic constitutive expressions for $\hat{\boldsymbol{v}}^{\mathrm{S}}(\bullet), \hat{\boldsymbol{v}}_{\boldsymbol{l}}^{\mathrm{S}}(\bullet)$, and $\hat{\bar{\tau}}(\bullet)$ have been established. For the explicit specification of these three functions, we propose first that the composition of the state space $\mathcal{Z}$ is composed of the four mesoscopic variables: $\boldsymbol{l}, \hat{\rho}^{\mathrm{S}}(\bullet), \nabla \hat{\rho}^{\mathrm{S}}(\bullet)$ and $\nabla_{l} \hat{\rho}^{\mathrm{S}}(\bullet)$, and the four macroscopic variables: temperature $\theta$, magnetic flux $\boldsymbol{B}^{\mathrm{S}}(\boldsymbol{x}, t)$, rate of deformation tensor $\boldsymbol{D}^{\mathrm{S}}(\boldsymbol{x}, t)\left(=\frac{1}{2}\left(\boldsymbol{\nabla} \boldsymbol{v}^{\mathrm{S}}+\left(\boldsymbol{\nabla} \boldsymbol{v}^{\mathrm{S}}\right)^{\mathrm{T}}\right)\right)$, and second moment of 
the distribution function $\mathrm{A}_{2}(\boldsymbol{x}, t)$, i.e.

$$
\mathcal{Z}=\left\{\boldsymbol{l}, \hat{\rho}^{\mathrm{S}}(\bullet), \nabla \hat{\rho}^{\mathrm{S}}(\bullet), \nabla_{l} \hat{\rho}^{\mathrm{S}}(\bullet), \theta(\boldsymbol{x}, t), \boldsymbol{B}^{\mathrm{S}}(\boldsymbol{x}, t), \boldsymbol{D}^{\mathrm{S}}(\boldsymbol{x}, t), \mathrm{A}_{2}(\boldsymbol{x}, t)\right\} .
$$

The macroscopic variables $\theta, \boldsymbol{B}^{\mathrm{S}}$, and $\boldsymbol{D}^{\mathrm{S}}$ are used to account for the influences of temperature, magnetic field and flow on the mesoscopic functions. The variable $\mathrm{A}_{2}$ is included to serve as internal variable in the constitutive consideration. Second, from the definition of $\hat{\boldsymbol{v}}_{l}^{\mathrm{S}}(\bullet)$ in equation (3.3) and by requiring that the distributions of $\boldsymbol{l}$ and $\boldsymbol{- l}$ be equal, the mesoscopic functions $\hat{\boldsymbol{v}}^{\mathrm{S}}(\bullet)$ and $\hat{\boldsymbol{v}}_{\boldsymbol{l}}^{\mathrm{S}}(\bullet)$ are then constrained by the symmetry conditions

$$
\hat{\boldsymbol{v}}^{\mathrm{S}}(-\boldsymbol{l})=\hat{\boldsymbol{v}}^{\mathrm{S}}(\boldsymbol{l}), \quad \hat{\boldsymbol{v}}_{\boldsymbol{l}}^{\mathrm{S}}(-\boldsymbol{l})=-\hat{\boldsymbol{v}}_{l}^{\mathrm{S}}(\boldsymbol{l}),
$$

which manifest that $\hat{\boldsymbol{v}}^{\mathrm{S}}(\bullet)$ is an even function of $\boldsymbol{l}$ and $\hat{\boldsymbol{v}}_{\boldsymbol{l}}^{\mathrm{S}}(\bullet)$ is an odd one. Hence, equation (6.11) will serve as a guiding rule to determine the expressions for the constitutive functions, $\hat{\boldsymbol{v}}^{\mathrm{S}}(\bullet)$ and $\hat{\boldsymbol{v}}_{l}^{\mathrm{S}}(\bullet)$. Two special cases of the second moment equation are now considered.

Case (i): for analytic convenience, we only take into account the linear relation in $l$ by assuming that the constitutive functions, which satisfy the symmetry conditions in equation (6.11), are in the form of

$$
\begin{gathered}
\hat{\boldsymbol{v}}_{l}^{\mathrm{S}}(\bullet)=\alpha_{1} \nabla_{l} \ln \hat{\boldsymbol{\rho}}^{\mathrm{S}}(\bullet)+\boldsymbol{l} \cdot\left(\alpha_{2} \mathrm{~A}_{2}+\alpha_{3} \boldsymbol{B}^{\mathrm{S}} \boldsymbol{B}^{\mathrm{S}}+\alpha_{4} \boldsymbol{D}^{\mathrm{S}}\right) \\
=\alpha_{1} \nabla_{l} \ln \hat{\boldsymbol{\rho}}^{\mathrm{S}}(\bullet)+\boldsymbol{l} \cdot \boldsymbol{C}(\boldsymbol{x}, t), \\
\hat{\boldsymbol{v}}^{\mathrm{S}}(\bullet)=\beta \nabla \ln \hat{\boldsymbol{\rho}}^{\mathrm{S}}(\bullet), \\
\hat{\bar{\tau}}(\bullet)=0 .
\end{gathered}
$$

In equation (6.12) we have set $\boldsymbol{C}=\boldsymbol{C}^{\mathrm{T}}=\alpha_{2} \mathrm{~A}_{2}+\alpha_{3} \boldsymbol{B}^{\mathrm{S}} \boldsymbol{B}^{\mathrm{S}}+\alpha_{4} \boldsymbol{D}^{\mathrm{S}}$. The parameters $\alpha_{1}, \alpha_{2}, \alpha_{3}, \alpha_{4}$ and $\beta$ are assumed to be functions of the temperature $\theta$. By inserting the constitutive functions (6.12), (6.13) and (6.14) into (6.10), it follows that

$$
\begin{aligned}
& \frac{\mathrm{d}_{\mathrm{S}}}{\mathrm{d} t} \mathrm{~A}_{2}(\boldsymbol{x}, t)+\int_{\mathcal{M}} \boldsymbol{l l} \nabla_{l} \cdot\left[\alpha_{1} \nabla_{l} \hat{f}(\bullet)+\hat{f}(\bullet) \boldsymbol{l} \cdot \boldsymbol{C}\right] \mathrm{d} v_{\mathcal{M}}+\nabla \cdot\left(\beta \nabla \mathrm{A}_{2}+\beta \nabla \ln \rho^{\mathrm{S}}(\boldsymbol{x}, t) \mathrm{A}_{2}\right) \\
& +\beta\left(\nabla \ln \rho^{\mathrm{S}}(\boldsymbol{x}, t) \cdot \nabla \mathrm{A}_{2}+\mathrm{A}_{2} \nabla \ln \rho^{\mathrm{S}}(\boldsymbol{x}, t) \cdot \nabla \ln \rho^{\mathrm{S}}(\boldsymbol{x}, t)\right) \\
& =\nabla \cdot\left(\boldsymbol{v}^{\mathrm{S}}(\boldsymbol{x}, t) \mathrm{A}_{2}\right)+\mathrm{A}_{2} \boldsymbol{v}^{\mathrm{S}}(\boldsymbol{x}, t) \cdot \nabla \ln \rho^{\mathrm{S}}(\boldsymbol{x}, t) .
\end{aligned}
$$

For more detailed manipulation of equation (6.15), we have the following reformulations:

$$
\begin{gathered}
\frac{\mathrm{d}_{\mathrm{S}}}{\mathrm{d} t} \mathbf{A}_{2}-\nabla \cdot\left(\boldsymbol{v}^{\mathrm{S}} \mathbf{A}_{2}\right)-\mathbf{A}_{2} \boldsymbol{v}^{\mathrm{S}} \cdot \nabla \ln \rho^{\mathrm{S}}=\frac{\partial}{\partial t} \mathbf{A}_{2}+\mathrm{A}_{2} \frac{\partial \ln \rho^{\mathrm{S}}}{\partial t}, \\
\int_{\mathcal{M}}\left(\nabla_{l}^{2} \hat{f}(\bullet)\right) \boldsymbol{l} \boldsymbol{l} \mathrm{d} v_{\mathcal{M}}=\int_{\mathcal{M}} \hat{f}(\bullet) \nabla_{l}^{2} \boldsymbol{l l} \mathrm{d} v_{\mathcal{M}}=2 \boldsymbol{l}, \\
\int_{\mathcal{M}} \nabla_{l} \cdot(\hat{f}(\bullet) \boldsymbol{l} \cdot \boldsymbol{C}) \boldsymbol{l l} \mathrm{d} v_{\mathcal{M}}=-\int_{\mathcal{M}} \hat{f}(\bullet) \boldsymbol{l} \cdot \boldsymbol{C} \cdot \nabla_{l}(\boldsymbol{l l}) \mathrm{d} v_{\mathcal{M}} \\
=-\boldsymbol{C}: \int_{\mathcal{M}} \hat{f}(\bullet) \boldsymbol{l}\left[\boldsymbol{l} \boldsymbol{l}+(\mid \boldsymbol{l})^{\mathrm{T}}\right] \mathrm{d} v_{\mathcal{M}}=-\left(\boldsymbol{C} \cdot \mathrm{A}_{2}+\mathrm{A}_{2} \cdot \boldsymbol{C}\right),
\end{gathered}
$$


where $\mathbf{I}$ is the identity tensor and condition (5.3) that the boundary of $\mathcal{M}$ is null has been used. In addition, by the mesoscopic average of $\hat{\boldsymbol{v}}^{\mathrm{S}}(\bullet)$ and from equations (3.16) and (6.13), we obtain

$$
\begin{gathered}
\boldsymbol{v}^{\mathrm{S}}=\beta \nabla \ln \rho^{\mathrm{S}}, \\
\frac{\partial \ln \rho^{\mathrm{S}}}{\partial t}=-\frac{\beta}{\rho^{\mathrm{S}}} \nabla^{2} \rho^{\mathrm{S}}=-\beta\left(\nabla^{2} \ln \rho^{\mathrm{S}}+\nabla \ln \rho^{\mathrm{S}} \cdot \nabla \ln \rho^{\mathrm{S}}\right) .
\end{gathered}
$$

Then, in view of equations (6.16)-(6.20), equation (6.15) can be further simplified to

$$
\frac{\partial}{\partial t} \mathrm{~A}_{2}+2 \alpha_{1} \mathrm{I}-\left(\boldsymbol{C} \cdot \mathrm{A}_{2}+\mathrm{A}_{2} \cdot \boldsymbol{C}\right)+\beta\left(\nabla^{2} \mathrm{~A}_{2}+2 \nabla \ln \rho^{\mathrm{S}} \cdot \mathrm{A}_{2}\right)=\mathbf{0},
$$

which is an evolution equation for the second-order internal variable $A_{2}$ on the base of the mesoscopic constitutive requirements (6.12), (6.13) and (6.14). This is different from the evolution equation of internal variable obtained from the macroscopic internal variable theory (Maugin \& Muschik 1994a,b), in which, besides the macroscopic constitutive requirements, the major restriction on this equation is the second law of thermodynamics.

Case (ii): if the higher-order terms involving $l l l, \nabla \nabla \hat{\rho}^{\mathrm{S}}(\bullet)$ and $\nabla \nabla \mathrm{A}_{2}$ are also considered, we assume constitutive functions, which still satisfy the symmetry conditions in equation (6.11), are in the form of

$$
\begin{gathered}
\hat{\boldsymbol{v}}_{l}^{\mathrm{S}}(\bullet)=\alpha_{1} \nabla_{l} \ln \hat{\boldsymbol{\rho}}^{\mathrm{S}}(\bullet) \\
+\boldsymbol{l} \cdot\left(\alpha_{2} \mathrm{~A}_{2}+\alpha_{3} \boldsymbol{B}^{\mathrm{S}} \boldsymbol{B}^{\mathrm{S}}+\alpha_{4} \boldsymbol{D}^{\mathrm{S}}+\alpha_{5} \frac{\nabla \nabla \hat{\boldsymbol{\rho}}^{\mathrm{S}}(\bullet)}{\hat{\boldsymbol{\rho}}^{\mathrm{S}}(\bullet)}+\alpha_{6} \boldsymbol{l l}: \mathrm{A}_{2} \mathrm{~A}_{2}+\alpha_{7} l \boldsymbol{l}: \nabla \nabla \mathrm{A}_{2}\right), \\
\hat{\boldsymbol{v}}^{\mathrm{S}}(\bullet)=\boldsymbol{v}^{\mathrm{S}}(\boldsymbol{x}, t), \\
\hat{\bar{\tau}}(\bullet)=0 .
\end{gathered}
$$

Then by inserting equations (6.22), (6.23) and (6.24) into (6.10) and after a simple calculation, we have

$$
\begin{aligned}
\frac{\mathrm{d}_{\mathrm{S}}}{\mathrm{d} t} \mathbf{A}_{2}(\boldsymbol{x}, t)= & 2 \alpha_{1} \mathbf{I}-2\left(\boldsymbol{C} \cdot \mathbf{A}_{2}\right)^{\mathrm{sym}}-\frac{2 \alpha_{5}}{\rho^{\mathrm{S}}(\boldsymbol{x}, t)}\left(\nabla \nabla \cdot\left(\mathbf{A}_{2} \rho^{\mathrm{S}}\right)\right)^{\mathrm{sym}} \\
& -2 \alpha_{6}\left(\mathbf{A}_{2}^{\mathrm{T}} \cdot\left(\mathbf{A}_{4}: \mathrm{A}_{2}\right)\right)^{\mathrm{sym}}-2 \alpha_{7}\left(\mathbf{A}_{4}: \cdot \nabla \nabla \mathrm{A}_{2}\right)^{\mathrm{sym}}
\end{aligned}
$$

where the superscript sym represents the symmetric part and the operator ' $:$ ' in $\mathrm{A}_{4}: \cdot \nabla \nabla \mathrm{A}_{2}$ means $\left(\mathbf{A}_{4}\right)_{i j k l} \frac{\partial}{\partial x_{i}} \frac{\partial}{\partial x_{j}}\left(\mathbf{A}_{2}\right)_{k m}$. In equation (6.25) it can be seen that the higher-order terms with $l l l$ will induce the higher-order moment $\mathrm{A}_{4}=\int \hat{f}(\bullet) l l l l \mathrm{~d} v_{\mathcal{M}}$ in the second moment equation of the mesoscopic distribution function. For this second moment equation including higher-order moment, a cut-off procedure or a closure relation will be required for the solvability of this equation (Papenfuss 2004). 


\section{Conclusions}

The macroscopic-mesoscopic concept is presented in this paper to study the response of MR materials. This twofold mixture represents a macroscopic solidfluid continuum, where the solid continuum, which is endowed with the internal structure due to the arrangement of particles, is a delicate mesoscopic mixture composed of different components with different mesoscopic variables $\boldsymbol{l}$ 's. Since these variables perform jumps in orientation with time, this introduces a production of orientational mass that affects the balance of evolution equation for the orientation mass function in the solid micro-continuum. The mesoscopic mass balance gives rise to an equation of the distribution function $\hat{f}(\bullet)$, and the role of the production term in this equation is analogous to that of the collision term in the Boltzmann equation. With the multiplication of this equation by $\boldsymbol{l}_{\alpha_{1}} \cdots \boldsymbol{l}_{\alpha_{m}}$ and then with the integration over the manifold $\mathcal{M}$, one immediately obtains the macroscopic $m$ th moment equation for the mesoscopic distribution function. Two special cases are briefly studied by adequately specifying the expressions of the mesoscopic constitutive functions $\hat{\boldsymbol{v}}_{\boldsymbol{l}}^{\mathrm{S}}(\bullet), \hat{\boldsymbol{v}}^{\mathrm{S}}(\bullet)$ and $\hat{\bar{\tau}}(\bullet)$.

The mesoscopic concept used in this paper mainly follows the one proposed by Blenk et al. (1991a) and their subsequent works on liquid crystals. However, four extensions are made compared with Blenk et al.'s works. First, we have applied the mesoscopic concept to the modelling of MR fluids. Once the mesoscopic variable $\boldsymbol{l}$ was defined, the procedure to derive the balance equations of liquid crystals made by the above research group could be adopted to establish the balance equations of the solid continuum of MR materials. The other three extensions then follow: (i) the mesoscopic variable $\boldsymbol{l}$ adopted in this paper is a man-made vector and it could be discontinuous in time, which does not occur for the director of liquid crystals; (ii) the concept of macroscopic-mesoscopic mixture is introduced so that the mesoscopic mixture becomes merely the solid component of the MR material and (iii) the magnetic field is not only included as a parameter in the derivation, but it also plays the key role to create the mesoscopic $\boldsymbol{l}$ field.

The mesoscopic approach provides more fundamental knowledge of the behaviour of materials with internal structure. For MR materials with magneticbased structures, the stress $\mathrm{t}^{\mathrm{S}}(\boldsymbol{x}, t)$ in equation (5.13) expressed by this approach is implicitly relevant to the magnetic field since, according to the definition of $\boldsymbol{l}$ in $\S 3$, no such vector $\boldsymbol{l}$ could be defined while the magnetic field is turned off. As for the determination of the magnitude of magnetic dependence of stress, more systematic mesoscopic constitutive information about $\hat{\rho}^{\mathrm{S}}(\bullet), \hat{\boldsymbol{v}}^{\mathrm{S}}(\bullet)$ and $\hat{\boldsymbol{t}}(\bullet)$ is definitely required.

The role played by the vector $\boldsymbol{l}$ is similar to that played by the velocity vector $\boldsymbol{c}$ in the Boltzmann equation. Since the appearance of $\boldsymbol{c}$ in the phase density of the Boltzmann equation gives an evolution of the particle distribution, consideration of $\boldsymbol{l}$ in the mesoscopic distribution provides information of the arrangement of the solid particles of MR fluids. Moreover, even through the originality of the mesoscopic equation for the distribution function (6.6) is different from that of the Boltzmann equation, the similarity of both equations such as $\boldsymbol{l}-\boldsymbol{c}$ correspondence and production-collision analogy provides an extension of the study of this mesoscopic theory to an unexplored realm that deserves future study. 
The author thanks Professor Kolumban Hutter for many helpful and instructive comments. This work was supported by the R. O. C. National Science Council under grants NSC-94-2211-E-002-075, NSC-93-2211-E-260-007 and NSC-92-2211-E-002-085.

\section{References}

Ashour, O., Rogers, C. A. \& Kordonsky, W. 1996 Magnetorheological fluids: materials, characterization, and devices. J. Intell. Mater. Syst. Struct. 7, 123-130.

Blenk, S. \& Muschik, W. 1991 Orientational balances for nematic liquid crystals. J. Non-Equilib. Thermodyn. 16, 67-87.

Blenk, S., Ehrentraut, H. \& Muschik, W. 1991a Statistical foundation of macroscopic balances for liquid crystals in alignment tensor formulation. Physica A 174, 119-138. (doi:10.1016/03784371(91)90420-H)

Blenk, S., Ehrentraut, H. \& Muschik, W. $1991 b$ Orientation-balances for liquid crystals and their representation by alignment tensors. Mol. Cryst. Liq. Cryst. 204, 133-141.

Blenk, S., Ehrentraut, H. \& Muschik, W. 1992 Macroscopic constitutive equations for liquid crystals induced by their mesoscopic orientation distribution. Int. J. Eng. Sci. 30, 1127-1143. (doi:10.1016/0020-7225(92)90062-L)

Block, H. \& Kelly, J. P. 1988 Review article: electrorheology. J. Appl. D Appl. Phys. 21, 1661-1677. (doi:10.1088/0022-3727/21/12/001)

Bowen, R. M. 1976 Theory of mixtures. In Continuum physics III (ed. A. C. Eringen), pp. 1-127. New York: Academic Press.

Chen, K. C. \& Yeh, C. S. $2002 a$ A mixture model for magneto-rheological materials. Continuum Mech. Thermodyn. 15, 495-510. (doi:10.1007/s001610200085)

Chen, K. C. \& Yeh, C. S. $2002 b$ Description of magnetorheological behavior with internal variables. Int. J. Eng. Sci. 40, 461-482. (doi:10.1016/S0020-7225(01)00074-X)

de Groot, S. R. \& Suttorp, L. G. 1972 Foundations of electrodynamics. Amsterdam: NorthHolland.

Edwards, S. F. \& Grinev, D. V. 2001 Transmission of stress in granular materials as a problem of statistical mechanics. Physica A 302, 162-186. (doi:10.1016/S0378-4371(01)00462-9)

Ehrentraut, H., Muschik, W. \& Papenfuss, C. 1997 Mesoscopically derived orientation dynamics of liquid crystals. J. Non-Equilib. Thermodyn. 22, 285-298.

Eringen, A. C. \& Maugin, G. A. 1990 Electrodynamics of continua I,II. New York: Springer.

Faria, S. H. 2001 Mixtures with continuous diversity: general theory and application to polymer solutions. Continuum Mech. Thermodyn. 13, 91-120. (doi:10.1007/s001610100043)

Faria, S. H. \& Hutter, K. 2002 A systematic approach to the thermodynamics of single and mixed flowing media with microstructure. Part I. Balance equations and jump conditions. Continuum Mech. Thermodyn. 14, 459-481. (doi:10.1007/s001610200084)

Hutter, K. \& Jöhnk, K. 2004 Continuum methods of physical modeling. Berlin: Springer.

Hutter, K. \& van de Ven, A. A. F. 1978 Field matter interaction in thermoelastic solids. New York: Springer.

Jackson, J. D. 1975 Classical electrodynamics. New York: Wiley.

Liu, I.-S. 2002 Continuum mechanics. Berlin: Springer.

Maugin, G. A. \& Muschik, W. 1994a Thermodynamics with internal variables. Part I. General concepts. J. Non-Equilib. Thermodyn. 19, 217-249.

Maugin, G. A. \& Muschik, W. $1994 b$ Thermodynamics with internal variables. Part II. Applications. J. Non-Equilib. Thermodyn. 19, 250-289.

Müller, I. 1985 Thermodynamics. New York: Pitman.

Müller, I. \& Ruggeri, T. 1998 Rational extended thermodynamics, 2nd edn. New York: Springer/ Pitman.

Muschik, W. \& Su, B. 1997 Mesoscopic interpretation of Fokker-Planck equation describing time behavior of liquid crystal orientaion. J. Chem. Phys. 107, 580-584. (doi:10.1063/1.474418) 
Muschik, W., Ehrentraut, H. \& Blenk, S. 1995 Ericksen-Leslie liquid crystal theory revisited from a mesoscopic point of view. J. Non-Equilib. Thermodyn. 20, 92-101.

Muschik, W., Ehrentraut, H. \& Papenfuss, C. 2000 Concepts of mesoscopic continuum physics with application to biaxial liquid crystals. J. Non-Equilib. Thermodyn. 25, 179-197. (doi:10. 1515/JNETDY.2000.011)

Muschik, W., Papenfuss, C. \& Ehrentraut, H. 2004 Mesoscopic theory of liquid crystals. J. NonEquilib. Thermodyn. 29, 75-106. (doi:10.1515/JNETDY.2004.006)

Nahmad-Molinari, Y., Arancibia-Bulnes, C. A. \& Ruiz-Suárez, J. C. 1999 Sound in magnetorheological slurry. Phys. Rev. Lett. 82, 727-730. (doi:10.1103/PhysRevLett.82.727)

Papenfuss, C. 2000 Theory of liquid crystals as an example of mesoscopic continuum mechanics. Comput. Mater. Sci. 19, 45-52. (doi:10.1016/S0927-0256(00)00138-5)

Papenfuss, C. 2004 A closure relation for the higher order alignment tensors in liquid crystal theory and the alignment-fabric tensors in damage mechanics from a statistical background. Physics $A$ 331, 23-41. (doi:10.1016/S0378-4371(03)00551-X)

Papenfuss, C. \& Muschik, W. 1995 Constitutive theory for two dimensional liquid crystals. Mol. Cryst. Liq. Cryst. 262, 473-484.

Papenfuss, C., Ván, P. \& Muschik, W. 2003 Mesoscopic theory of microcracks. Arch. Mech. 55, 459-477.

Truesdell, C. 1984 Rational thermodynamics, 2nd edn. New York: Springer.

Ván, P., Papenfuss, C. \& Muschik, W. 2000 Mesoscopic dynamics of microcracks. Phys. Rev. E 62, 6206-6215.

Yeh, C. S. \& Chen, K. C. 1997 A thermodynamic model for magnetorheological fluids. Continuum Mech. Thermodyn. 9, 273-291. (doi:10.1007/s001610050071) 